\title{
Oscillation criteria for third-order functional half-linear dynamic equations
}

Taher S Hassan ${ }^{1,2^{*}}$, Ravi P Agarwal ${ }^{3,4}$ and Wael W Mohammed ${ }^{1,2}$

\section{"Correspondence:}

tshassan@mans.edu.eg

'Department of Mathematics,

Faculty of Science, University of Hail,

Hail, 2440, Saudi Arabia

${ }^{2}$ Department of Mathematics,

Faculty of Science, Mansoura University, Mansoura, 35516, Egypt

Full list of author information is

available at the end of the article

\begin{abstract}
In this paper, we study the third-order functional dynamic equation

$$
\left\{r_{2}(t) \phi_{\alpha_{2}}\left(\left[r_{1}(t) \phi_{\alpha_{1}}\left(x^{\Delta}(t)\right)\right]^{\Delta}\right)\right\}^{\Delta}+q(t) \phi_{\alpha}(x(g(t)))=0,
$$

on an upper-unbounded time scale $\mathbb{T}$. We will extend the so-called Hille and Nehari type criteria to third-order dynamic equations on time scales. This work extends and improves some known results in the literature on third-order nonlinear dynamic equations and the results are established for a time scale $\mathbb{T}$ without assuming certain restrictive conditions on $\mathbb{T}$. Some examples are given to illustrate the main results.
\end{abstract}

MSC: 34K11;39A10; 39A99; 34N05

Keywords: oscillation criteria; third order; dynamic equations; time scales

\section{Introduction}

We are concerned with the oscillatory behavior of the third-order half-linear functional dynamic equation

$$
\left\{r_{2}(t) \phi_{\alpha_{2}}\left(\left[r_{1}(t) \phi_{\alpha_{1}}\left(x^{\Delta}(t)\right)\right]^{\Delta}\right)\right\}^{\Delta}+q(t) \phi_{\alpha}(x(g(t)))=0
$$

on an upper-unbounded time scale $\mathbb{T}$, where $\phi_{\alpha}(u):=|u|^{\alpha-1} u, \alpha_{1}, \alpha_{2}, \alpha:=\alpha_{1} \alpha_{2}>0 ; r_{i}$, $i=1,2$, are positive rd-continuous functions on $\mathbb{T}$ such that, for $t_{0} \in \mathbb{T}$,

$$
\int_{t_{0}}^{\infty} r_{i}^{-\frac{1}{\alpha_{i}}}(t) \Delta t=\infty
$$

$q$ is a positive rd-continuous function on $\mathbb{T}$; and $g: \mathbb{T} \rightarrow \mathbb{T}$ is a rd-continuous function such that $\lim _{t \rightarrow \infty} g(t)=\infty$. Throughout this paper, we let

$$
x^{[i]}:=r_{i} \phi_{\alpha_{i}}\left(\left[x^{[i-1]}\right]^{\Delta}\right), \quad i=1,2 \text {, with } x^{[0]}=x .
$$

We will assume that the reader is familiar with the basic facts of time scales and time scale notation, for an excellent introduction to the calculus on time scales, see Bohner and Peterson [1, 2]. By a solution of equation (1.1) we mean a nontrivial real-valued function $x \in C_{\mathrm{rd}}^{1}\left[T_{x}, \infty\right)_{\mathbb{T}}$ for some $T_{x} \geq t_{0}$ for a positive constant $t_{0} \in \mathbb{T}$ such that

(c) The Author(s) 2017. This article is distributed under the terms of the Creative Commons Attribution 4.0 International License (http://creativecommons.org/licenses/by/4.0/), which permits unrestricted use, distribution, and reproduction in any medium, provided you give appropriate credit to the original author(s) and the source, provide a link to the Creative Commons license, and indicate if changes were made. 
$x^{[1]}(t), x^{[2]}(t) \in C_{\mathrm{rd}}^{1}\left[T_{x}, \infty\right)_{\mathbb{T}}$ and $x(t)$ satisfies equation $(1.1)$ on $\left[T_{x}, \infty\right)_{\mathbb{T}}$, where $C_{\mathrm{rd}}$ is the space of right-dense continuous functions.

In the following, we state some oscillation results for differential equations that will be related to our oscillation results for (1.1) on time scales and explain the important contributions of this paper. In 1918, Fite [3] studied the oscillatory behavior of solutions of the second-order linear differential equation

$$
x^{\prime \prime}(t)+q(t) x(t)=0
$$

and showed that if

$$
\int_{t_{0}}^{\infty} q(s) d s=\infty
$$

then every solution of equation (1.4) is oscillatory. Hille [4] improved the condition (1.5) and showed that if

$$
\liminf _{t \rightarrow \infty} t \int_{t}^{\infty} q(s) d s>\frac{1}{4},
$$

then every solution of (1.4) is oscillatory. Nehari [5] proved that if

$$
\liminf _{t \rightarrow \infty} \frac{1}{t} \int_{t_{0}}^{t} s^{2} q(s) d s>\frac{1}{4}
$$

then every solution of (1.4) is oscillatory. Wong [6] generalized the Hille-type condition (1.6) for the delay equation

$$
x^{\prime \prime}(t)+q(t) x(g(t))=0
$$

where $g(t) \geq k t$ with $0<k<1$, and proved that if

$$
\liminf _{t \rightarrow \infty} t \int_{t}^{\infty} q(s) d s>\frac{1}{4 k},
$$

then every solution of (1.8) is oscillatory. Erbe [7] improved the condition (1.9) and proved that if

$$
\liminf _{t \rightarrow \infty} t \int_{t}^{\infty} q(s) \frac{g(s)}{s} d s>\frac{1}{4},
$$

then every solution of $(1.8)$ is oscillatory where $g(t) \leq t$. Ohriska [8] proved that, if

$$
\limsup _{t \rightarrow \infty} t \int_{t}^{\infty} q(s) \frac{g(s)}{s} d s>1,
$$

then every solution of (1.8) is oscillatory.

Erbe, Peterson and Saker [9] established Hille and Nehari oscillation criteria for the third-order dynamic equation

$$
x^{\Delta \Delta \Delta}(t)+q(t) x(t)=0
$$


where $q$ is a positive real-valued rd-continuous function on $\mathbb{T}$, we list the main results of [9] as follows.

Theorem 1.1 ([9]) Every solution of equation (1.12) is either oscillatory or tends to zero eventually provided that

$$
\int_{t_{0}}^{\infty} \int_{z}^{\infty} \int_{u}^{\infty} q(s) \Delta s \Delta u \Delta z=\infty
$$

holds and one of the following conditions is satisfied:

(a)

$$
\liminf _{t \rightarrow \infty} \int_{t}^{\infty} \frac{h_{2}\left(s, t_{0}\right)}{\sigma(s)} q(s) \Delta s>\frac{1}{4}
$$

(b)

$$
\liminf _{t \rightarrow \infty} \frac{1}{t} \int_{t_{0}}^{t} h_{2}\left(s, t_{0}\right) \sigma(s) q(s) \Delta s>\frac{l^{*}}{1+l^{*}}
$$

where $l^{*}:=\limsup _{t \rightarrow \infty} \frac{\sigma(t)}{t}$ and $h_{2}(t, s)$ is the Taylor monomial of degree 2; see [1], Section 1.6.

Erbe, Hassan and Peterson [10] studied the third-order dynamic equation

$$
\left(r_{2}(t)\left[\left(r_{1}(t) x^{\Delta}(t)\right)^{\Delta}\right]^{\alpha}\right)^{\Delta}+q(t) x^{\alpha}(g(t))=0,
$$

where $\alpha$ is a quotient of odd positive integers, one of which we give below.

Theorem 1.2 ([10]) Every solution of equation (1.15) is either oscillatory or tends to zero eventually provided that

$$
g(t) \geq t, \quad r_{1}^{\Delta}(t) \geq 0,
$$

and

$$
\int_{t_{0}}^{\infty} \frac{1}{r_{1}(z)} \int_{z}^{\infty}\left[\frac{1}{r_{2}(u)} \int_{u}^{\infty} q(s) \Delta s\right]^{1 / \alpha} \Delta u \Delta z=\infty,
$$

hold, and one of the following conditions is satisfied:

(a)

$$
\liminf _{t \rightarrow \infty} \frac{t^{\alpha}}{r_{1}^{\alpha}(t)} \int_{\sigma(t)}^{\infty} q(s) \Delta s>\frac{\alpha^{\alpha}}{l^{\alpha^{2}}(\alpha+1)^{\alpha+1}}
$$

(b)

$$
\liminf _{t \rightarrow \infty} \frac{t^{\alpha}}{r_{1}^{\alpha}(t)} \int_{\sigma(t)}^{\infty} q(s) \Delta s+\liminf _{t \rightarrow \infty} \frac{1}{t} \int_{t_{0}}^{t} \frac{s^{\alpha+1}}{r_{1}^{\alpha}(s)} q(s) \Delta s>\frac{1}{l^{\alpha(\alpha+1)}},
$$

where $l:=\liminf _{t \rightarrow \infty} \frac{t}{\sigma(t)}$. 
Saker [11] considered dynamic equation (1.1) with $\alpha_{1}=r_{1}=1$ and $\alpha=\alpha_{2}$ is a quotient of odd positive integers when $g(t) \leq t$, namely,

$$
\left\{r_{2}(t)\left[x^{\Delta \Delta}(t)\right]^{\alpha_{2}}\right\}^{\Delta}+q(t) x^{\alpha}(g(t))=0 .
$$

He established some Hille and Nehari type oscillation criteria for (1.17), one of which we give below.

Theorem 1.3 ([11], Theorem 3.4 and Corollaries 3.3, 3.4) Every solution of equation (1.17) is either oscillatory or tends to zero eventually provided that

$$
g(t) \leq t, \quad r_{2}^{\Delta}(t) \geq 0
$$

and

$$
\int_{t_{0}}^{\infty} \int_{z}^{\infty}\left[\frac{1}{r_{2}(u)} \int_{u}^{\infty} q(s) \Delta s\right]^{1 / \alpha} \Delta u \Delta z=\infty
$$

hold, and one of the following conditions is satisfied:

(a)

$$
\liminf _{t \rightarrow \infty} \frac{t^{\alpha}}{r_{2}(t)} \int_{\sigma(t)}^{\infty} A(s) \Delta s>\frac{\alpha^{\alpha}}{l^{\alpha^{2}}(\alpha+1)^{\alpha+1}}
$$

(b)

$$
\liminf _{t \rightarrow \infty} \frac{t^{\alpha}}{r_{2}(t)} \int_{\sigma(t)}^{\infty} A(s) \Delta s+\liminf _{t \rightarrow \infty} \frac{1}{t} \int_{t_{0}}^{t} \frac{s^{\alpha+1}}{r_{2}(s)} A(s) \Delta s>\frac{1}{l^{\alpha(\alpha+1)}}
$$

(c)

$$
\liminf _{t \rightarrow \infty} \frac{1}{t} \int_{t_{0}}^{t} \frac{s^{\alpha+1}}{r_{2}(s)} A(s) \Delta s>\frac{1}{l^{\alpha(\alpha+1)}}
$$

(d)

$$
\begin{gathered}
\liminf _{t \rightarrow \infty} \frac{t^{\alpha}}{r_{2}(t)} \int_{\sigma(t)}^{\infty} A(s) \Delta s>\frac{1}{l^{\alpha(\alpha+1)}}, \\
\text { where } A(s):=q(s)\left(\frac{h_{2}\left(g(s), t_{0}\right)}{\sigma(s)}\right)^{\alpha} \text { and } l:=\liminf _{t \rightarrow \infty} \frac{t}{\sigma(t)} .
\end{gathered}
$$

Theorem 1.4 ([11], Corollary 3.5) Assume that (1.19) holds with $r_{2}(t)=1$ and $\alpha=\alpha_{2}=1$. Assume

$$
\liminf _{t \rightarrow \infty} t \int_{\sigma(t)}^{\infty} q(s)\left(\frac{h_{2}\left(g(s), t_{0}\right)}{\sigma(s)}\right)^{\alpha} \Delta s>\frac{1}{4 l} .
$$

Every solution of the equation

$$
x^{\Delta \Delta \Delta}(t)+q(t) x(g(t))=0,
$$

is either oscillatory or tends to zero eventually. 
As a special case when $g(t)=t,(1.20)$ reduces to

$$
\liminf _{t \rightarrow \infty} t \int_{\sigma(t)}^{\infty} q(s)\left(\frac{h_{2}\left(s, t_{0}\right)}{\sigma(s)}\right)^{\alpha} \Delta s>\frac{1}{4 l} .
$$

Comparing (1.14) with (1.21) reveals that the results in [9] improve that of [11] for equation (1.12).

Wang and $\mathrm{Xu}$ in [12] considered the third-order dynamic equation

$$
\left(r_{2}(t)\left[\left(r_{1}(t) x^{\Delta}(t)\right)^{\Delta}\right]^{\alpha}\right)^{\Delta}+q(t) x(t)=0,
$$

where $\alpha \geq 1$ is a quotient of odd positive integers and with the condition

$$
\lim _{t \rightarrow \infty} \frac{\bar{R}(\sigma(t))}{\bar{R}(t)}=1,
$$

where

$$
\begin{aligned}
& R_{2}\left(t, t_{1}\right):=\int_{t_{1}}^{t} r_{2}^{-\frac{1}{\alpha}}(s) \Delta s, \quad R_{3}\left(t, t_{1}\right):=\int_{t_{1}}^{t} \frac{R_{2}\left(s, t_{1}\right)}{r_{1}(s)} \Delta s, \\
& r(t):=\frac{\alpha}{r_{1}(t)} R_{2}\left(t, t_{1}\right) R_{3}^{\alpha-1}\left(t, t_{1}\right) \quad \text { and } \quad \bar{R}(t):=\int_{t_{1}}^{t} r(s) \Delta s .
\end{aligned}
$$

Note that (1.22) depends on a concrete time scale. Very recently, Agarwal, Bohner, Li, and Zhang [13] extended the Hille and Nehari oscillation criteria to the third-order delay dynamic equation

$$
\left(r_{2}(t)\left(r_{1}(t) x^{\Delta}(t)\right)^{\Delta}\right)^{\Delta}+q(t) x(g(t))=0
$$

where $g(t) \leq t$ on $\left[t_{0}, \infty\right)_{\mathbb{T}}$. The results in [13] included the results which were established in [9] and without condition (1.22). For more results on dynamic equations, we refer the reader to $[12,14-30]$.

The purpose of this paper is to derive some Hille and Nehari oscillation criteria to the more general third-order dynamic equation (1.1) with Laplacians and deviating argument on a general time scale and without assuming the conditions (1.16), (1.18) and (1.22). The results in this paper improve the results in [9-13] for third-order dynamic equations and for both cases $g(t) \leq t$ or $g(t) \geq t$.

This paper is organized as follows: After this Introduction, we present our main results in Section 2, followed by demonstrating examples. All the proofs of the main results are given in Sections 3 and 4.

\section{Main results}

In this section we present the following oscillation criteria of (1.1). The first result is a Fite-Wintner type oscillation criterion.

Theorem 2.1 Assume that (1.2) and

$$
\int_{t_{0}}^{\infty} q(s) \Delta s=\infty
$$

Then every solution of equation (1.1) is either oscillatory or tends to zero eventually. 
From Theorem 2.1, we can assume in the next theorems that $\int_{t_{0}}^{\infty} q(s) \Delta s<\infty$. In the following, we introduce the following notations:

$$
L:=\limsup _{t \rightarrow \infty}\left[\frac{R_{1}(\sigma(t))}{R_{1}(t)}\right]^{\alpha} \text { and } R_{i}(t):=\int_{t_{0}}^{t} r_{i}^{-\frac{1}{\alpha_{i}}}(s) \Delta s, \quad i=1,2 \text {, }
$$

and

$$
\varphi(t):= \begin{cases}1, & g(t) \geq t \\ {\left[\frac{R(g(t))}{R(t)}\right]^{\alpha},} & g(t) \leq t\end{cases}
$$

with

$$
R(t):=R_{2}^{-1 / \alpha_{1}}(t) R_{3}(t) \quad \text { and } \quad R_{3}(t):=\int_{t_{0}}^{t}\left[\frac{R_{2}(s)}{r_{1}(s)}\right]^{\frac{1}{\alpha_{1}}} \Delta s .
$$

Note that $1 \leq L \leq \infty$. Throughout this paper we assume that $L<\infty$.

Theorem 2.2 Assume that (1.2) and

$$
\int_{t_{0}}^{\infty} r_{1}^{-\frac{1}{\alpha_{1}}}(u)\left\{\int_{u}^{\infty} r_{2}^{-\frac{1}{\alpha_{2}}}(v)\left[\int_{v}^{\infty} q(s) \Delta s\right]^{\frac{1}{\alpha_{2}}} \Delta v\right\}^{\frac{1}{\alpha_{1}}} \Delta u=\infty .
$$

If

$$
\int_{t_{0}}^{\infty} \varphi(s) q(s) \Delta s=\infty
$$

then every solution of equation (1.1) is either oscillatory or tends to zero eventually.

Example 2.1 Consider the nonlinear third-order advanced dynamic equation

$$
\left\{t^{\alpha_{2}-1} \phi_{\alpha_{2}}\left(\left[t^{\alpha_{1}-1} \phi_{\alpha_{1}}\left(x^{\Delta}(t)\right)\right]^{\Delta}\right)\right\}^{\Delta}+\frac{1}{t^{1-\beta}} \phi_{\alpha}(x(g(t)))=0, \quad g(t) \geq t,
$$

where $\beta$ is a positive constant. Here $r_{i}(t)=t^{\alpha_{i}-1} 1, i=1,2$ and $q(t)=\frac{1}{t^{1-\beta}}$, then the conditions (1.2) and (2.3) hold since

$$
\int_{t_{0}}^{\infty} r_{i}^{-\frac{1}{\alpha_{i}}}(t) \Delta t=\int_{t_{0}}^{\infty} \frac{\Delta t}{t^{1-\frac{1}{\alpha_{i}}}}=\infty, \quad i=1,2,
$$

and

$$
\int_{t_{0}}^{\infty} \varphi(s) q(s) \Delta s=\int_{t_{0}}^{\infty} \frac{\Delta s}{s^{1-\beta}}=\infty,
$$

by Example 5.60 in [1]. Then by Theorem 2.2, every solution of (2.4) is oscillatory or tends to zero eventually.

From Theorem 2.2, we can assume in the next theorems that $\int_{t_{0}}^{\infty} \varphi(s) q(s) \Delta s<\infty$. 
Masan et al. Advances in Difference Equations ( 2017) 2017:111

Page 7 of 28

Theorem 2.3 Assume that (1.2) and (2.2) hold. If

$$
\liminf _{t \rightarrow \infty} R_{1}^{\alpha}(t) \int_{\sigma(t)}^{\infty} \varphi(s) q(s) \Delta s>\frac{(\alpha L)^{\alpha}}{(\alpha+1)^{\alpha+1}}
$$

then every solution of equation (1.1) is either oscillatory or tends to zero eventually.

Example 2.2 Consider the nonlinear third-order advanced dynamic equation

$$
\left\{t^{\alpha_{2}-1} \phi_{\alpha_{2}}\left(\left[\phi_{\alpha_{1}}\left(x^{\Delta}(t)\right)\right]^{\Delta}\right)\right\}^{\Delta}+\frac{\eta \alpha}{l t^{\alpha+1}} \phi_{\alpha}(x(g(t)))=0, \quad g(t) \geq t
$$

where $\eta$ is a positive constant and $l=\liminf _{t \rightarrow \infty}\left(\frac{t}{\sigma(t)}\right)^{\alpha}>0$. Here $r_{1}(t)=1, r_{2}(t)=t^{\alpha_{2}}$ and $q(t)=\frac{\eta \alpha}{l t^{\alpha+1}}$, then the condition (1.2) and (2.2) hold since

$$
\int_{t_{0}}^{\infty} r_{1}^{-\frac{1}{\alpha_{1}}}(t) \Delta t=\int_{t_{0}}^{\infty} \Delta t=\infty \quad \text { and } \quad \int_{t_{0}}^{\infty} r_{2}^{-\frac{1}{\alpha_{2}}}(t) \Delta t=\int_{t_{0}}^{\infty} \frac{\Delta t}{t}=\infty
$$

by Example 5.60 in [1] and

$$
\begin{aligned}
& \int_{t_{0}}^{\infty} r_{1}^{-\frac{1}{\alpha_{1}}}(u)\left\{\int_{u}^{\infty} r_{2}^{-\frac{1}{\alpha_{2}}}(v)\left[\int_{v}^{\infty} q(s) \Delta s\right]^{\frac{1}{\alpha_{2}}} \Delta v\right\}^{\frac{1}{\alpha_{1}}} \Delta u \\
& =\left(\frac{\eta}{l}\right)^{\frac{1}{\alpha}} \int_{t_{0}}^{\infty}\left\{\int_{u}^{\infty} \frac{1}{v}\left[\int_{v}^{\infty} \frac{\alpha}{s^{\alpha+1}} \Delta s\right]^{\frac{1}{\alpha_{2}}} \Delta v\right\}^{\frac{1}{\alpha_{1}}} \Delta u \\
& \geq\left(\frac{\eta}{l}\right)^{\frac{1}{\alpha}} \int_{t_{0}}^{\infty}\left\{\int_{u}^{\infty} \frac{1}{v}\left[\int_{v}^{\infty}\left(\frac{-1}{s^{\alpha}}\right)^{\Delta} \Delta s\right]^{\frac{1}{\alpha_{2}}} \Delta v\right\}^{\frac{1}{\alpha_{1}}} \Delta u \\
& =\left(\frac{\eta}{l}\right)^{\frac{1}{\alpha}} \int_{t_{0}}^{\infty}\left\{\int_{u}^{\infty} \frac{1}{v^{\alpha_{1}+1}} \Delta v\right\}^{\frac{1}{\alpha_{1}}} \Delta u \\
& \geq\left(\frac{\eta}{l}\right)^{\frac{1}{\alpha}} \int_{t_{0}}^{\infty}\left\{\frac{1}{\alpha_{1}} \int_{u}^{\infty}\left(\frac{-1}{v^{\alpha_{1}}}\right)^{\Delta} \Delta v\right\}^{\frac{1}{\alpha_{1}}} \Delta u \\
& \geq\left(\frac{\eta}{l}\right)^{\frac{1}{\alpha}} \frac{1}{\alpha_{1}^{\alpha_{1}}} \int_{t_{0}}^{\infty} \frac{\Delta u}{u}=\infty .
\end{aligned}
$$

Also

$$
\begin{aligned}
\liminf _{t \rightarrow \infty} R_{1}^{\alpha}(t) \int_{\sigma(t)}^{\infty} \varphi(s) q(s) \Delta s & =\frac{\eta}{l} \liminf _{t \rightarrow \infty} R_{1}^{\alpha}(t) \int_{\sigma(t)}^{\infty} \frac{\alpha \Delta s}{s^{\alpha+1}} \\
& \geq \frac{\eta}{l} \liminf _{t \rightarrow \infty} R_{1}^{\alpha}(t) \int_{\sigma(t)}^{\infty}\left(\frac{-1}{s^{\alpha}}\right)^{\Delta} \Delta s \\
& =\frac{\eta}{l} \liminf _{t \rightarrow \infty}\left(\frac{t}{\sigma(t)}-\frac{t_{0}}{\sigma(t)}\right)^{\alpha}>\frac{(\alpha L)^{\alpha}}{(\alpha+1)^{\alpha+1}}
\end{aligned}
$$

if $\eta>\frac{(\alpha L)^{\alpha}}{(\alpha+1)^{\alpha+1}}$. Then, by Theorem 2.3, every solution of (2.6) is oscillatory or tends to zero eventually if $\eta>\frac{(\alpha L)^{\alpha}}{(\alpha+1)^{\alpha+1}}$. 
Theorem 2.4 Assume that (1.2) and (2.2) hold. If for sufficiently large $T \in\left[t_{0}, \infty\right)_{\mathbb{T}}$,

$$
\liminf _{t \rightarrow \infty} \frac{1}{R_{1}(t)} \int_{T}^{t} R_{1}^{\alpha+1}(s) \varphi(s) q(s) \Delta s>L^{\alpha+1}\left(1-\frac{1}{(\alpha+1) L}\right),
$$

then every solution of equation (1.1) is either oscillatory or tends to zero eventually.

Remark 2.1 If the assumption (2.2) is not satisfied, we have some sufficient conditions which ensure that every solution $x(t)$ of (1.1) oscillates or $\lim _{t \rightarrow \infty} x(t)$ exists (finite).

Example 2.3 Consider the nonlinear third-order delay dynamic equation

$$
\left\{r_{2}(t) \phi_{\alpha_{2}}\left(\left[r_{1}(t) \phi_{\alpha_{1}}\left(x^{\Delta}(t)\right)\right]^{\Delta}\right)\right\}^{\Delta}+\frac{\eta r_{1}^{-1 / \alpha_{1}}(t)}{\varphi(t) R_{1}^{\alpha+1}(t)} \phi_{\alpha}(x(g(t)))=0, \quad g(t) \leq t,
$$

where $\eta$ is a positive constant. Choose $r_{i}$ and $\alpha_{i}, i=1,2$, satisfying (1.2). To see that (2.7) holds note that

$$
\begin{aligned}
\liminf _{t \rightarrow \infty} \frac{1}{R_{1}(t)} \int_{T}^{t} R_{1}^{\alpha+1}(s) \varphi(s) q(s) \Delta s & =\eta \liminf _{t \rightarrow \infty} \frac{1}{R_{1}(t)} \int_{T}^{t} r_{1}^{-1 / \alpha_{1}}(t) \Delta s \\
& =\eta \liminf _{t \rightarrow \infty}\left(1-\frac{R_{1}(T)}{R_{1}(t)}\right)=\eta .
\end{aligned}
$$

By Theorem 2.4 and Remark 2.1, every solution $x(t)$ of (2.8) is oscillatory or $\lim _{t \rightarrow \infty} x(t)$ exists if $\eta>L^{\alpha+1}\left(1-\frac{1}{(\alpha+1) L}\right) \geq \frac{\alpha}{\alpha+1}$.

Remark 2.2 The important point to note here is that the recent results due to [9-13] and others do not apply to equations (2.4), (2.6) and (2.8).

Theorem 2.5 Assume that $0<\alpha \leq 1$ and (1.2), and (2.2) hold. If for sufficiently large $T \in$ $\left[t_{0}, \infty\right)_{\mathbb{T}}$,

$$
\liminf _{t \rightarrow \infty} \frac{1}{R_{1}(t)} \int_{T}^{t} R_{1}^{\alpha+1}(s) \varphi(s) q(s) \Delta s>L^{\alpha+1}\left(1-\frac{1}{\alpha+L}\right)
$$

then every solution of equation (1.1) is either oscillatory or tends to zero eventually.

Theorem 2.6 Assume that $\alpha \geq 1$ and (1.2), and (2.2) hold. If for sufficiently large $T \in$ $\left[t_{0}, \infty\right)_{\mathbb{T}}$,

$$
\liminf _{t \rightarrow \infty} \frac{1}{R_{1}(t)} \int_{T}^{t} R_{1}^{\alpha+1}(s) \varphi(s) q(s) \Delta s>\frac{\alpha L^{\alpha+2}}{1+\alpha L},
$$

then every solution of equation (1.1) is either oscillatory or tends to zero eventually.

The next result is an Ohriskais type oscillation criterion.

Theorem 2.7 Assume that (1.2) and (2.2) hold. If

$$
\limsup _{t \rightarrow \infty} R_{3}^{\alpha}(t) \int_{t}^{\infty} \varphi(s) q(s) \Delta s>1
$$

then every solution of equation (1.1) is either oscillatory or tends to zero eventually. 
In the following we state further oscillation criteria for equation (1.1).

Theorem 2.8 Every solution of equation (1.1) is either oscillatory or tends to zero eventually provided that (1.2) and (2.2) hold and one of the following conditions is satisfied:

(a)

$$
\int_{t_{0}}^{\infty} \bar{\varphi}(s) q(s) \Delta s=\infty
$$

(b)

$$
\liminf _{t \rightarrow \infty} R_{1}^{\alpha}(t) \int_{\sigma(t)}^{\infty} \bar{\varphi}(s) q(s) \Delta s>\frac{(\alpha L)^{\alpha}}{(\alpha+1)^{\alpha+1}}
$$

(c)

$$
\liminf _{t \rightarrow \infty} \frac{1}{R_{1}(t)} \int_{T}^{t} R_{1}^{\alpha+1}(s) \bar{\varphi}(s) q(s) \Delta s>L^{\alpha+1}\left(1-\frac{1}{(\alpha+1) L}\right) ;
$$

(d)

$$
\begin{aligned}
& \liminf _{t \rightarrow \infty} \frac{1}{R_{1}(t)} \int_{T}^{t} R_{1}^{\alpha+1}(s) \bar{\varphi}(s) q(s) \Delta s>L^{\alpha+1}\left(1-\frac{1}{\alpha+L}\right) \quad \text { if } 0<\alpha \leq 1, \\
& \liminf _{t \rightarrow \infty} \frac{1}{R_{1}(t)} \int_{T}^{t} R_{1}^{\alpha+1}(s) \bar{\varphi}(s) q(s) \Delta s>\frac{\alpha L^{\alpha+2}}{1+\alpha L} \quad \text { if } \alpha \geq 1,
\end{aligned}
$$

for sufficiently large $T \in\left[t_{0}, \infty\right)_{\mathbb{T}}$, where

$$
\bar{\varphi}(t):= \begin{cases}1, & g(t) \geq \sigma(t), \\ {\left[\frac{R(g(t))}{R(\sigma(t))}\right]^{\alpha},} & g(t) \leq \sigma(t) .\end{cases}
$$

Theorem 2.9 Every solution of equation (1.1) is either oscillatory or tends to zero eventually provided hat (1.2) and (2.2) hold and one of the following conditions is satisfied:

(a)

$$
\int_{t_{0}}^{\infty} \hat{\varphi}(s) q(s) \Delta s=\infty
$$

(b)

$$
\liminf _{t \rightarrow \infty} R_{2}^{\alpha_{2}}(t) \int_{\sigma(t)}^{\infty} \hat{\varphi}(s) q(s) \Delta s>\frac{\left(\alpha_{2} \hat{L}\right)^{\alpha_{2}}}{\left(\alpha_{2}+1\right)^{\alpha_{2}+1}}
$$

(c)

$$
\liminf _{t \rightarrow \infty} \frac{1}{R_{2}(t)} \int_{T}^{t} R_{2}^{\alpha_{2}+1}(s) \hat{\varphi}(s) q(s) \Delta s>\hat{L}^{\alpha_{2}+1}\left(1-\frac{1}{\left(\alpha_{2}+1\right) \hat{L}}\right)
$$


(d)

$$
\begin{aligned}
& \liminf _{t \rightarrow \infty} \frac{1}{R_{2}(t)} \int_{T}^{t} R_{2}^{\alpha_{2}+1}(s) \hat{\varphi}(s) q(s) \Delta s>\hat{L}^{\alpha_{2}+1}\left(1-\frac{1}{\alpha_{2}+\hat{L}}\right) \quad \text { if } 0<\alpha_{2} \leq 1, \\
& \liminf _{t \rightarrow \infty} \frac{1}{R_{2}(t)} \int_{T}^{t} R_{2}^{\alpha_{2}+1}(s) \hat{\varphi}(s) q(s) \Delta s>\frac{\alpha_{2} \hat{L}^{\alpha_{2}+2}}{1+\alpha_{2} \hat{L}} \quad \text { if } \alpha_{2} \geq 1,
\end{aligned}
$$

for sufficiently large $T \in\left[t_{0}, \infty\right)_{\mathbb{T}}$, where

$$
\hat{\varphi}(t):= \begin{cases}R^{\alpha}(t), & g(t) \geq t, \\ R^{\alpha}(g(t)), & g(t) \leq t .\end{cases}
$$

and assume that $\hat{L}:=\limsup _{t \rightarrow \infty}\left[\frac{R_{2}(\sigma(t))}{R_{2}(t)}\right]^{\alpha_{2}}<\infty$.

\section{Technical lemmas}

In this section we prove the following lemmas which will play an important role in the proof of our main results.

Lemma 3.1 Let (1.2) holds. If $x(t)$ is an eventually positive solution of equation (1.1), then we only have the following two cases:

(I) $x^{[1]}(t)>0, x^{[2]}(t)>0,\left(x^{[2]}(t)\right)^{\Delta}<0$;

(II) $x^{[1]}(t)<0, x^{[2]}(t)>0,\left(x^{[2]}(t)\right)^{\Delta}<0$,

eventually.

Lemma 3.2 Let $x(t)$ be an eventually positive solution of (1.1) satisfying (II) of Lemma 3.1. If (2.2) holds, then $\lim _{t \rightarrow \infty} x(t)=0$.

Lemma 3.3 Let $x(t)$ be an eventually positive solution of (1.1) satisfying (I) of Lemma 3.1. Then

$$
\int_{t_{0}}^{\infty} q(s) \Delta s<\infty
$$

Proof Without loss of generality, assume that

$$
x(g(t))>0, \quad x^{[1]}(t)>0, \quad x^{[2]}(t)>0, \quad\left[x^{[2]}(t)\right]^{\Delta}<0 \quad \text { on }\left[t_{0}, \infty\right)_{\mathbb{T}} .
$$

Integrating both sides of the dynamic equation (1.1) from $t_{0}$ to $t \in\left[t_{0}, \infty\right)_{\mathbb{T}}$, we obtain

$$
x^{[2]}\left(t_{0}\right) \geq x^{[2]}\left(t_{0}\right)-x^{[2]}(t)=\int_{t_{0}}^{t} q(s) x^{\alpha}(g(s)) \Delta s .
$$

Since $x^{\Delta}(t)>0$, then $x(t) \geq x\left(t_{0}\right):=c>0$ for $t \geq t_{0}$ and so there exists $t_{1} \in\left[t_{0}, \infty\right)_{\mathbb{T}}$ such that $g(t) \in\left[t_{0}, \infty\right)_{\mathbb{T}}$ and $x(g(t)) \geq c$ for $t \geq t_{1}$. It follows that

$$
x^{[2]}\left(t_{0}\right) \geq c^{\alpha} \int_{t_{0}}^{t} q(s) \Delta s,
$$

which implies (3.1). 
Lemma 3.4 Let $x(t)$ be an eventually positive solution of (1.1) satisfying (I) of Lemma 3.1.

Then

$$
x(t) \geq \phi_{\alpha}^{-1}\left[x^{[2]}(t)\right] R_{3}(t) \quad \text { for } t \in\left[t_{0}, \infty\right)_{\mathbb{T}}
$$

and

$$
\left[\frac{x(t)}{R(t)}\right]^{\Delta}<0 \quad \text { for } t \in\left(t_{0}, \infty\right)_{\mathbb{T}}
$$

Proof Without loss of generality, assume that

$$
x(g(t))>0, \quad x^{[1]}(t)>0, \quad x^{[2]}(t)>0, \quad\left[x^{[2]}(t)\right]^{\Delta}<0 \quad \text { on }\left[t_{0}, \infty\right)_{\mathbb{T}} .
$$

By using the fact that $x^{[2]}$ is strictly decreasing on $\left[t_{0}, \infty\right)_{\mathbb{T}}$. Then, for $t \in\left[t_{0}, \infty\right)_{\mathbb{T}}$,

$$
\begin{aligned}
x^{[1]}(t) & \geq x^{[1]}(t)-x^{[1]}\left(t_{0}\right)=\int_{t_{0}}^{t} \phi_{\alpha_{2}}^{-1}\left[x^{[2]}(s)\right] r_{2}^{-\frac{1}{\alpha_{2}}}(s) \Delta s \\
& \geq \phi_{\alpha_{2}}^{-1}\left[x^{[2]}(t)\right] \int_{t_{0}}^{t} r_{2}^{-\frac{1}{\alpha_{2}}}(s) \Delta s=\phi_{\alpha_{2}}^{-1}\left[x^{[2]}(t)\right] R_{2}(t),
\end{aligned}
$$

which implies that

$$
x^{\Delta}(t) \geq \phi_{\alpha}^{-1}\left[x^{[2]}(t)\right]\left[\frac{R_{2}(t)}{r_{1}(t)}\right]^{\frac{1}{\alpha_{1}}}
$$

where $\alpha=\alpha_{1} \alpha_{2}$. In the same way, we have

$$
\begin{aligned}
x(t) & \geq \phi_{\alpha}^{-1}\left[x^{[2]}(t)\right] \int_{t_{0}}^{t}\left[\frac{R_{2}(s)}{r_{1}(s)}\right]^{\frac{1}{\alpha_{1}}} \Delta s \\
& =\phi_{\alpha}^{-1}\left[x^{[2]}(t)\right] R_{3}(t) .
\end{aligned}
$$

From (3.2), we note that

$$
\left[\frac{x^{[1]}(t)}{R_{2}(t)}\right]^{\Delta}=\frac{r_{2}^{-1 / \alpha_{2}}(t)}{R_{2}(t) R_{2}(\sigma(t))}\left[\phi_{\alpha_{2}}^{-1}\left[x^{[2]}(t)\right] R_{2}(t)-x^{[1]}(t)\right],
$$

we have

$$
\left[\frac{x^{[1]}(t)}{R_{2}(t)}\right]^{\Delta}<0 \quad \text { for } t \in\left(t_{0}, \infty\right)_{\mathbb{T}} .
$$

Then

$$
\begin{aligned}
x(t) & \geq x(t)-x\left(t_{0}\right)=\int_{t_{0}}^{t} \phi_{\alpha_{1}}^{-1}\left[x^{[1]}(s)\right] r_{1}^{-\frac{1}{\alpha_{1}}}(s) \Delta s \\
& =\int_{t_{0}}^{t} \phi_{\alpha_{1}}^{-1}\left[\frac{x^{[1]}(s)}{R_{2}(s)}\right]\left[\frac{R_{2}(s)}{r_{1}(s)}\right]^{\frac{1}{\alpha_{1}}} \Delta s
\end{aligned}
$$




$$
\begin{aligned}
& \geq \phi_{\alpha_{1}}^{-1}\left[\frac{x^{[1]}(t)}{R_{2}(t)}\right] \int_{t_{0}}^{t}\left[\frac{R_{2}(s)}{r_{1}(s)}\right]^{\frac{1}{\alpha_{1}}} \Delta s \\
& =\phi_{\alpha_{1}}^{-1}\left[x^{[1]}(t)\right] R(t),
\end{aligned}
$$

which yields

$$
\left[\frac{x(t)}{R(t)}\right]^{\Delta}<0 \quad \text { for } t \in\left(t_{0}, \infty\right)_{\mathbb{T}} .
$$

This completes the proof.

Lemma 3.5 Let $x(t)$ be an eventually positive solution of (1.1) satisfying (I) of Lemma 3.1.

Then

$$
\int_{t_{0}}^{\infty} \varphi(s) q(s) \Delta s<\infty
$$

Proof Without loss of generality, assume

$$
\begin{aligned}
& x(t)>0, \quad x(g(t))>0, \\
& x^{[1]}(t)>0, \quad x^{[2]}(t)>0, \quad\left(x^{[2]}(t)\right)^{\Delta}<0 \quad \text { on }\left[t_{0}, \infty\right)_{\mathbb{T}} .
\end{aligned}
$$

Let $t \in\left[t_{0}, \infty\right)_{\mathbb{T}}$ be fixed. If $g(t) \geq t$, then $x(g(t)) \geq x(t)$ by the fact that $x$ is strictly increasing. Now we consider the case when $g(t) \leq t$. In view of Lemma 3.4 there exists $t_{1} \in\left[t_{0}, \infty\right)_{\mathbb{T}}$ such that $g(t)>t_{0}$ and

$$
x(g(t)) \geq \frac{R(g(t))}{R(t)} x(t) \quad \text { for } t \geq t_{1} .
$$

In both cases, from the definition of $\varphi(t)$, equation (1.1) becomes

$$
\left[x^{[2]}(t)\right]^{\Delta}+\varphi(t) q(t) \phi_{\alpha}(x(t)) \leq 0 .
$$

Integrating both sides of the above inequality from $t_{1}$ to $t \in\left[t_{1}, \infty\right)_{\mathbb{T}}$, we obtain

$$
x^{[2]}\left(t_{1}\right) \geq x^{[2]}\left(t_{1}\right)-x^{[2]}(t)=\int_{t_{1}}^{t} \varphi(s) q(s) x^{\alpha}(s) \Delta s .
$$

Since $x^{\Delta}(t)>0$, then $x(t) \geq x\left(t_{1}\right):=c>0$ for $t \geq t_{1}$, then

$$
x^{[2]}\left(t_{1}\right) \geq c^{\alpha} \int_{t_{0}}^{t} \varphi(s) q(s) \Delta s,
$$

which implies (3.3). This completes the proof.

Lemma 3.6 Let $x(t)$ be an eventually positive solution of (1.1) satisfying (I) of Lemma 3.1. Then, for $t \geq T$,

$$
w^{\Delta}(t) \leq-\varphi(t) q(t)-\alpha r_{1}^{-1 / \alpha_{1}}(t) w^{1+\frac{1}{\alpha}}(\sigma(t)),
$$


and

$$
w^{\Delta}(t) \leq-\bar{\varphi}(t) q(t)-\alpha r_{1}^{-1 / \alpha_{1}}(t) w^{1+\frac{1}{\alpha}}(\sigma(t))
$$

for sufficiently large $T \in\left[t_{0}, \infty\right)_{\mathbb{T}}$, where

$$
w(t):=\frac{x^{[2]}(t)}{x^{\alpha}(t)}
$$

Proof Without loss of generality, assume that

$$
x(g(t))>0, \quad x^{[1]}(t)>0, \quad x^{[2]}(t)>0, \quad\left[x^{[2]}(t)\right]^{\Delta}<0 \quad \text { on }\left[t_{0}, \infty\right)_{\mathbb{T}} .
$$

Using the product rule and the quotient rule, we get

$$
\begin{aligned}
w^{\Delta}(t) & =\left(\frac{x^{[2]}(t)}{x^{\alpha}(t)}\right)^{\Delta}=\frac{1}{x^{\alpha}(t)}\left[x^{[2]}(t)\right]^{\Delta}+\left(\frac{1}{x^{\alpha}(t)}\right)^{\Delta} x^{[2]}(\sigma(t)) \\
& =\frac{\left[x^{[2]}(t)\right]^{\Delta}}{x^{\alpha}(t)}-\frac{\left(x^{\alpha}(t)\right)^{\Delta}}{x^{\alpha}(t) x^{\alpha}(\sigma(t))} x^{[2]}(\sigma(t)) .
\end{aligned}
$$

From (1.1) and the definition of $w(t)$ we have

$$
w^{\Delta}(t)=-q(t)\left(\frac{x(g(t))}{x(t)}\right)^{\alpha}-\frac{\left(x^{\alpha}(t)\right)^{\Delta}}{x^{\alpha}(t)} w(\sigma(t)) .
$$

As shown in the proof of Lemma 3.5, there exists $t_{1} \in\left[t_{0}, \infty\right)_{\mathbb{T}}$ such that $g(t)>t_{0}$ and

$$
\left(\frac{x(g(t))}{x(t)}\right)^{\alpha} \geq \varphi(t) \quad \text { for } t \in\left[t_{1}, \infty\right)_{\mathbb{T}}
$$

Therefore

$$
w^{\Delta}(t) \leq-\varphi(t) q(t)-\frac{\left(x^{\alpha}(t)\right)^{\Delta}}{x^{\alpha}(t)} w(\sigma(t)) .
$$

By the Pötzsche chain rule ([1], Theorem 1.90), we obtain

$$
\begin{aligned}
\left(x^{\alpha}(t)\right)^{\Delta} & =\alpha\left(\int_{0}^{1}\left[x(t)+h \mu(t) x^{\Delta}(t)\right]^{\alpha-1} d h\right) x^{\Delta}(t) \\
& =\alpha\left(\int_{0}^{1}\left[(1-h) x(t)+h x^{\sigma}(t)\right]^{\alpha-1} d h\right) x^{\Delta}(t) \\
& \geq \begin{cases}\alpha\left(x^{\sigma}(t)\right)^{\alpha-1} x^{\Delta}(t), & 0<\alpha \leq 1, \\
\alpha x^{\alpha-1}(t) x^{\Delta}(t), & \alpha \geq 1 .\end{cases}
\end{aligned}
$$

If $0<\alpha \leq 1$, then

$$
w^{\Delta}(t)<-\varphi(t) q(t)-\alpha \frac{x^{\Delta}(t)}{x^{\sigma}(t)}\left(\frac{x^{\sigma}(t)}{x(t)}\right)^{\alpha} w(\sigma(t))
$$


and if $\alpha \geq 1$, then

$$
w^{\Delta}(t) \leq-\varphi(t) q(t)-\alpha \frac{x^{\Delta}(t)}{x^{\sigma}(t)} \frac{x^{\sigma}(t)}{x(t)} w(\sigma(t)) .
$$

Note that $x(t)$ is strictly increasing on $\left[t_{1}, \infty\right)_{\mathbb{T}}$ we see that, for $\alpha>0$,

$$
w^{\Delta}(t) \leq-\varphi(t) q(t)-\alpha \frac{x^{\Delta}(t)}{x^{\sigma}(t)} w(\sigma(t))
$$

Since $x^{[2]}(t)$ is strictly decreasing on $\left[t_{1}, \infty\right)$ we obtain

$$
\begin{aligned}
x^{[1]}(t) & \geq x^{[1]}(t)-x^{[1]}\left(t_{1}\right)=\int_{t_{1}}^{t} \phi_{\alpha_{2}}^{-1}\left[x^{[2]}(s)\right] r_{2}^{-\frac{1}{\alpha_{2}}}(s) \Delta s \\
& \geq \phi_{\alpha_{2}}^{-1}\left[x^{[2]}(t)\right] \int_{t_{1}}^{t} r_{2}^{-\frac{1}{\alpha_{2}}}(s) \Delta s \geq \phi_{\alpha_{2}}^{-1}\left[x^{[2]}(\sigma(t))\right]\left(R_{2}(t)-R_{2}\left(t_{1}\right)\right),
\end{aligned}
$$

which implies

$$
\begin{aligned}
\frac{x^{\Delta}(t)}{x^{\sigma}(t)} & \geq \frac{\phi_{\alpha}^{-1}\left[x^{[2]}(\sigma(t))\right]}{x^{\sigma}(t)}\left[\frac{R_{2}(t)-R_{2}\left(t_{1}\right)}{r_{1}(t)}\right]^{1 / \alpha_{1}} \\
& =w^{1 / \alpha}(\sigma(t))\left[\frac{R_{2}(t)-R_{2}\left(t_{1}\right)}{r_{1}(t)}\right]^{1 / \alpha_{1}} .
\end{aligned}
$$

By (1.2), we can choose $t_{2} \geq t_{1}$ such that $R_{2}(t)-R_{2}\left(t_{1}\right) \geq 1$ for $t \geq t_{2}$, then, from (3.8) and (3.9), we have

$$
w^{\Delta}(t) \leq-\varphi(t) q(t)-\alpha r_{1}^{-1 / \alpha}(t) w^{1+\frac{1}{\alpha}}(\sigma(t)) \quad \text { for } t \geq t_{2} .
$$

Also by the quotient rule, we get

$$
\begin{aligned}
w^{\Delta}(t) & =\left(\frac{x^{[2]}(t)}{x^{\alpha}(t)}\right)^{\Delta}=\frac{\left[x^{[2]}(t)\right]^{\Delta}}{x^{\alpha}(\sigma(t))}-\frac{\left(x^{\alpha}(t)\right)^{\Delta} x^{[2]}(t)}{x^{\alpha}(t) x^{\alpha}(\sigma(t))} \\
& \leq \frac{\left[x^{[2]}(t)\right]^{\Delta}}{x^{\alpha}(\sigma(t))}-\frac{\left(x^{\alpha}(t)\right)^{\Delta} x^{[2]}(\sigma(t))}{x^{\alpha}(t) x^{\alpha}(\sigma(t))} .
\end{aligned}
$$

From (1.1) and the definition of $w(t)$ we have

$$
w^{\Delta}(t) \leq-q(t)\left(\frac{x(g(t))}{x(\sigma(t))}\right)^{\alpha}-\frac{\left(x^{\alpha}(t)\right)^{\Delta}}{x^{\alpha}(t)} w(\sigma(t)) .
$$

Analogously as in the proof of Lemma 3.5 , there exists $t_{1} \in\left[t_{0}, \infty\right)_{\mathbb{T}}$ such that $g(t)>t_{0}$ and

$$
\left(\frac{x(g(t))}{x(\sigma(t))}\right)^{\alpha} \geq \bar{\varphi}(t) \quad \text { for } t \in\left[t_{1}, \infty\right)_{\mathbb{T}} .
$$

Therefore

$$
w^{\Delta}(t) \leq-\bar{\varphi}(t) q(t)-\frac{\left(x^{\alpha}(t)\right)^{\Delta}}{x^{\alpha}(t)} w(\sigma(t)) .
$$


The rest of the argument is similar to that of previous results as $\varphi$ is replaced by $\bar{\varphi}$, and hence it is omitted.

We introduce the following notations:

$$
r_{*}:=\liminf _{t \rightarrow \infty} R_{1}^{\alpha}(t) w(\sigma(t)) \quad \text { and } \quad R_{*}:=\limsup _{t \rightarrow \infty} R_{1}^{\alpha}(t) w(\sigma(t))
$$

where $w$ is defined by (3.5). So for $\varepsilon>0$, then by the definitions of $r_{*}, R_{*}$ and $L$ we can pick $T \in\left[t_{0}, \infty\right)_{\mathbb{T}}$, sufficiently large, so that

$$
r_{*}-\varepsilon \leq R_{1}^{\alpha}(t) w(\sigma(t)) \leq R_{*}+\varepsilon \quad \text { and } \quad\left[\frac{R_{1}(\sigma(t))}{R_{1}(t)}\right]^{\alpha} \leq L+\varepsilon \quad \text { for } t \in[T, \infty)_{\mathbb{T}} .
$$

Lemma 3.7 Let $x(t)$ be an eventually positive solution of (1.1) satisfying (I) of Lemma 3.1.

Then

$$
\liminf _{t \rightarrow \infty} R_{1}^{\alpha}(t) \int_{\sigma(t)}^{\infty} \varphi(s) q(s) \Delta s \leq r_{*}-\frac{r_{*}^{1+\frac{1}{\alpha}}}{L} .
$$

Proof Integrating (3.4) from $\sigma(t) \geq T$ to $v \in[t, \infty)_{\mathbb{T}}$ and using the fact that $w>0$, we have

$$
\begin{aligned}
-w(\sigma(t)) & \leq w(v)-w(\sigma(t)) \\
& \leq-\int_{\sigma(t)}^{v} \varphi(s) q(s) \Delta s-\alpha \int_{\sigma(t)}^{v} r_{1}^{-1 / \alpha_{1}}(s) w^{1+\frac{1}{\alpha}}(\sigma(s)) \Delta s .
\end{aligned}
$$

Taking $v \rightarrow \infty$ we get

$$
-w(\sigma(t)) \leq-\int_{\sigma(t)}^{\infty} \varphi(s) q(s) \Delta s-\alpha \int_{\sigma(t)}^{\infty} r_{1}^{-1 / \alpha_{1}}(s) w^{1+\frac{1}{\alpha}}(\sigma(s)) \Delta s .
$$

Multiplying both sides of (3.11) by $R_{1}^{\alpha}(t)$, we obtain

$$
\begin{aligned}
-R_{1}^{\alpha}(t) w(\sigma(t)) \leq & -R_{1}^{\alpha}(t) \int_{\sigma(t)}^{\infty} \varphi(s) q(s) \Delta s \\
& -\alpha R_{1}^{\alpha}(t) \int_{\sigma(t)}^{\infty} r_{1}^{-1 / \alpha_{1}}(s) w^{1+\frac{1}{\alpha}}(\sigma(s)) \Delta s \\
= & -R_{1}^{\alpha}(t) \int_{\sigma(t)}^{\infty} \varphi(s) q(s) \Delta s \\
& -\alpha R_{1}^{\alpha}(t) \int_{\sigma(t)}^{\infty} \frac{R_{1}^{\Delta}(s)}{R_{1}^{\alpha+1}(s)}\left(R_{1}^{\alpha}(s) w(\sigma(s))\right)^{1+\frac{1}{\alpha}} \Delta s .
\end{aligned}
$$

Therefore, by using (3.10), we have

$$
\begin{aligned}
-R_{1}^{\alpha}(t) w(\sigma(t)) \leq & -R_{1}^{\alpha}(t) \int_{\sigma(t)}^{\infty} \varphi(s) q(s) \Delta s \\
& -\left(r_{*}-\varepsilon\right)^{1+\frac{1}{\alpha}} R_{1}^{\alpha}(t) \int_{\sigma(t)}^{\infty} \alpha \frac{R_{1}^{\Delta}(s)}{R_{1}^{\alpha+1}(s)} \Delta s .
\end{aligned}
$$


Using the Pötzsche chain rule ([1], Theorem 1.90), we get

$$
\begin{aligned}
\left(\frac{-1}{R_{1}^{\alpha}}\right)^{\Delta} & =\alpha \int_{0}^{1} \frac{1}{\left[R_{1}+h \mu(s) R_{1}^{\Delta}\right]^{\alpha+1}} d h R_{1}^{\Delta} \\
& \leq \alpha \frac{R_{1}^{\Delta}}{R_{1}^{\alpha+1}} .
\end{aligned}
$$

Then from (3.12) and (3.13), we have

$$
\begin{aligned}
-R_{1}^{\alpha}(t) w(t) & \leq-R_{1}^{\alpha}(t) \int_{\sigma(t)}^{\infty} \varphi(s) q(s) \Delta s-\left(r_{*}-\varepsilon\right)^{1+\frac{1}{\alpha}}\left[\frac{R_{1}(t)}{R_{1}(\sigma(t))}\right]^{\alpha} \\
& \leq-R_{1}^{\alpha}(t) \int_{\sigma(t)}^{\infty} \varphi(s) q(s) \Delta s-\frac{\left(r_{*}-\varepsilon\right)^{1+\frac{1}{\alpha}}}{L+\varepsilon}
\end{aligned}
$$

which yields

$$
R_{1}^{\alpha}(t) \int_{\sigma(t)}^{\infty} \varphi(s) q(s) \Delta s \leq R_{1}^{\alpha}(t) w(\sigma(t))-\frac{\left(r_{*}-\varepsilon\right)^{1+\frac{1}{\alpha}}}{L+\varepsilon}
$$

Taking the liminf of both sides as $t \rightarrow \infty$ we get

$$
\liminf _{t \rightarrow \infty} R_{1}^{\alpha}(t) \int_{\sigma(t)}^{\infty} \varphi(s) q(s) \Delta s \leq r_{*}-\frac{\left(r_{*}-\varepsilon\right)^{1+\frac{1}{\alpha}}}{L+\varepsilon} .
$$

Since $\varepsilon>0$ is arbitrary, we get the desired inequality:

$$
\liminf _{t \rightarrow \infty} R_{1}^{\alpha}(t) \int_{\sigma(t)}^{\infty} \varphi(s) q(s) \Delta s \leq r_{*}-\frac{r_{*}^{1+\frac{1}{\alpha}}}{L} .
$$

This completes the proof.

Lemma 3.8 Let $x(t)$ be an eventually positive solution of (1.1) satisfying (I) of Lemma 3.1. Then

$$
\liminf _{t \rightarrow \infty} \frac{1}{R_{1}(t)} \int_{T}^{t} R_{1}^{\alpha+1}(s) \varphi(s) q(s) \Delta s \leq L^{\alpha+1}-R_{*}
$$

for sufficiently large $T \in\left[t_{0}, \infty\right)_{\mathbb{T}}$.

Proof Multiplying both sides of (3.4) by $R_{1}^{\alpha+1}(t)$ and integrating from $T$ to $t \in[T, \infty)_{\mathbb{T}}$, we get

$$
\begin{aligned}
\int_{T}^{t} R_{1}^{\alpha+1}(s) w^{\Delta}(s) \Delta s \leq & -\int_{T}^{t} R_{1}^{\alpha+1}(s) \varphi(s) q(s) \Delta s \\
& -\alpha \int_{T}^{t} r_{1}^{-\frac{1}{\alpha_{1}}}(s)\left(R_{1}^{\alpha}(s) w(\sigma(s))\right)^{\frac{\alpha+1}{\alpha}} \Delta s
\end{aligned}
$$


Using integration by parts, we obtain

$$
\begin{aligned}
R_{1}^{\alpha+1}(t) w(t) \leq & R_{1}^{\alpha+1}(T) w(T)+\int_{T}^{t}\left[R_{1}^{\alpha+1}(s)\right]^{\Delta} w(\sigma(s)) \Delta s \\
& -\int_{T}^{t} R_{1}^{\alpha+1}(s) \varphi(s) q(s) \Delta s \\
& -\alpha \int_{T}^{t} r_{1}^{-\frac{1}{\alpha_{1}}}(s)\left(R_{1}^{\alpha}(s) w(\sigma(s))\right)^{\frac{\alpha+1}{\alpha}} \Delta s .
\end{aligned}
$$

By the Pötzsche chain rule, we get

$$
\begin{aligned}
{\left[R_{1}^{\alpha+1}(s)\right]^{\Delta} } & =(\alpha+1) \int_{0}^{1}\left[R_{1}(s)+h \mu(s) R_{1}^{\Delta}(s)\right]^{\alpha} d h r_{1}^{-1 / \alpha_{1}}(s) \\
& =(\alpha+1) \int_{0}^{1}\left[(1-h) R_{1}(s)+h R_{1}(\sigma(s))\right]^{\alpha} d h r_{1}^{-1 / \alpha_{1}}(s) \\
& \leq(\alpha+1) R_{1}^{\alpha}(\sigma(s)) r_{1}^{-1 / \alpha_{1}}(s) .
\end{aligned}
$$

Hence

$$
\begin{aligned}
R_{1}^{\alpha+1}(t) w(t) \leq & R_{1}^{\alpha+1}(T) w(T) \\
& +(\alpha+1) \int_{T}^{t} r_{1}^{-\frac{1}{\alpha_{1}}}(s)\left[\frac{R_{1}(\sigma(s))}{R_{1}(s)}\right]^{\alpha} R_{1}^{\alpha}(s) w(\sigma(s)) \Delta s \\
& -\int_{T}^{t} R_{1}^{\alpha+1}(s) \varphi(s) q(s) \Delta s-\alpha \int_{T}^{t} r_{1}^{-\frac{1}{\alpha_{1}}}(s)\left(R_{1}^{\alpha}(s) w(\sigma(s))\right)^{\frac{\alpha+1}{\alpha}} \Delta s
\end{aligned}
$$

By (3.10), we then get

$$
\begin{aligned}
R_{1}^{\alpha+1}(t) w(t) \leq & R_{1}^{\alpha+1}(T) w(T)-\int_{T}^{t} R_{1}^{\alpha+1}(s) \varphi(s) q(s) \Delta s \\
& +\int_{T}^{t} r_{1}^{-1 / \alpha_{1}}(s)\left[(\alpha+1)(L+\varepsilon) R_{1}^{\alpha}(s) w(\sigma(s))\right. \\
& \left.-\alpha\left(R_{1}^{\alpha}(s) w(\sigma(s))\right)^{\frac{\alpha+1}{\alpha}}\right] \Delta s .
\end{aligned}
$$

Using the inequality

$$
B u-A u^{\frac{\alpha+1}{\alpha}} \leq \frac{\alpha^{\alpha}}{(\alpha+1)^{\alpha+1}} \frac{B^{\alpha+1}}{A^{\alpha}}
$$

with $A=\alpha, B=(\alpha+1)(L+\varepsilon)$ and $u=R_{1}^{\alpha}(s) w(\sigma(s))$, we get

$$
R_{1}^{\alpha+1}(t) w(t) \leq R_{1}^{\alpha+1}(T) w(T)-\int_{T}^{t} R_{1}^{\alpha+1}(s) \varphi(s) q(s) \Delta s+(L+\varepsilon)^{\alpha+1} R_{1}(t)
$$

Dividing both sides by $R_{1}(t)$, we obtain

$$
R_{1}^{\alpha}(t) w(t) \leq \frac{R_{1}^{\alpha+1}(T) w(T)}{R_{1}(t)}-\frac{1}{R_{1}(t)} \int_{T}^{t} R_{1}^{\alpha+1}(s) \varphi(s) q(s) \Delta s+(L+\varepsilon)^{\alpha+1}
$$


Since $w^{\sigma}(t) \leq w(t)$, we get

$$
R_{1}^{\alpha}(t) w(\sigma(t)) \leq \frac{R_{1}^{\alpha+1}(T) w(T)}{R_{1}(t)}-\frac{1}{R_{1}(t)} \int_{T}^{t} R_{1}^{\alpha+1}(s) \varphi(s) q(s) \Delta s+(L+\varepsilon)^{\alpha+1}
$$

Taking the limsup of both sides as $t \rightarrow \infty$ we obtain

$$
R_{*} \leq-\liminf _{t \rightarrow \infty} \frac{1}{R_{1}(t)} \int_{T}^{t} R_{1}^{\alpha+1}(s) \varphi(s) q(s) \Delta s+(L+\varepsilon)^{\alpha+1}
$$

Since $\varepsilon>0$ is arbitrary, we get

$$
\liminf _{t \rightarrow \infty} \frac{1}{R_{1}(t)} \int_{T}^{t} R_{1}^{\alpha+1}(s) \varphi(s) q(s) \Delta s \leq L^{\alpha+1}-R_{*} .
$$

This completes the proof.

Lemma 3.9 Let $x(t)$ be an eventually positive solution of (1.1) satisfying (I) of Lemma 3.1.

Then

$$
\liminf _{t \rightarrow \infty} \frac{1}{R_{1}(t)} \int_{T}^{t} R_{1}^{\alpha+1}(s) \varphi(s) q(s) \Delta s \leq[(\alpha+1) L-1] R_{*}-\alpha r_{*}^{1+\frac{1}{\alpha}}
$$

for sufficiently large $T \in\left[t_{0}, \infty\right)_{\mathbb{T}}$.

Proof Multiplying both sides (3.4) by $R_{1}^{\alpha+1}(t)$, we get

$$
\begin{aligned}
R_{1}^{\alpha+1}(t) w^{\Delta}(t) \leq & -R_{1}^{\alpha+1}(t) \varphi(t) q(t)-\alpha r_{1}^{-1 / \alpha_{1}}(t) R_{1}^{\alpha+1}(t) w^{1+\frac{1}{\alpha}}(\sigma(t)) \\
= & -R_{1}^{\alpha+1}(t) \varphi(t) q(t) \\
& -\alpha r_{1}^{-1 / \alpha_{1}}(t) R_{1}^{\alpha}(t) w(\sigma(t)) R_{1}(t) w^{\frac{1}{\alpha}}(\sigma(t)) \\
\leq & -R_{1}^{\alpha+1}(t) \varphi(t) q(t)-\alpha r_{1}^{-1 / \alpha_{1}}(t)\left(r_{*}-\varepsilon\right)^{1+\frac{1}{\alpha}}
\end{aligned}
$$

Integrating the above inequality (3.16) from $T$ to $t \in[T, \infty)_{\mathbb{T}}$, we obtain

$$
\int_{T}^{t} R_{1}^{\alpha+1}(s) w^{\Delta}(s) \Delta s \leq-\int_{T}^{t} R_{1}^{\alpha+1}(s) \varphi(s) q(s) \Delta s-\alpha\left(r_{*}-\varepsilon\right)^{1+\frac{1}{\alpha}} \int_{T}^{t} r_{1}^{-1 / \alpha_{1}}(s) \Delta s .
$$

By integrating by parts, we obtain

$$
\begin{aligned}
R_{1}^{\alpha+1}(t) w(t) \leq & R_{1}^{\alpha+1}(T) w(T)+\int_{T}^{t}\left[R_{1}^{\alpha+1}(s)\right]^{\Delta} w(\sigma(s)) \Delta s \\
& -\int_{T}^{t} R_{1}^{\alpha+1}(s) \varphi(s) q(s) \Delta s-\alpha\left(r_{*}-\varepsilon\right)^{1+\frac{1}{\alpha}}\left[R_{1}(t)-R_{1}(T)\right]
\end{aligned}
$$

As seen in the proof of Lemma 3.8, we have

$$
\left[R_{1}^{\alpha+1}(s)\right]^{\Delta} \leq(\alpha+1) R_{1}^{\alpha}(\sigma(s)) r_{1}^{-1 / \alpha_{1}}(s) .
$$


Therefore

$$
\begin{aligned}
R_{1}^{\alpha+1}(t) w(t) \leq & R_{1}^{\alpha+1}(T) w(T) \\
& +(\alpha+1) \int_{T}^{t}\left[\frac{R_{1}(\sigma(s))}{R_{1}(s)}\right]^{\alpha} r_{1}^{-1 / \alpha_{1}}(s) R_{1}^{\alpha}(s) w(\sigma(s)) \Delta s \\
& -\int_{T}^{t} R_{1}^{\alpha+1}(s) \varphi(s) q(s) \Delta s-\alpha\left(r_{*}-\varepsilon\right)^{1+\frac{1}{\alpha}}\left[R_{1}(t)-R_{1}(T)\right] \\
\leq & R_{1}^{\alpha+1}(T) w(T)+(\alpha+1)(L+\varepsilon)\left(R_{*}+\varepsilon\right)\left[R_{1}(t)-R_{1}(T)\right] \\
& -\int_{T}^{t} R_{1}^{\alpha+1}(s) \varphi(s) q(s) \Delta s-\alpha\left(r_{*}-\varepsilon\right)^{1+\frac{1}{\alpha}}\left[R_{1}(t)-R_{1}(T)\right] .
\end{aligned}
$$

Dividing both sides by $R_{1}$, we have

$$
\begin{aligned}
R_{1}^{\alpha}(t) w(t) \leq & \frac{R_{1}^{\alpha+1}(T) w(T)}{R_{1}(t)}+(\alpha+1)(L+\varepsilon)\left(R_{*}+\varepsilon\right)\left[1-\frac{R_{1}(T)}{R_{1}(t)}\right] \\
& -\frac{1}{R_{1}(t)} \int_{T}^{t} R_{1}^{\alpha+1}(s) \varphi(s) q(s) \Delta s \\
& -\alpha\left(r_{*}-\varepsilon\right)^{1+\frac{1}{\alpha}}\left[1-\frac{R_{1}(T)}{R_{1}(t)}\right] .
\end{aligned}
$$

Taking the lim sup of both sides as $t \rightarrow \infty$ and using (1.2), we get

$$
\begin{aligned}
R_{*} \leq & (\alpha+1)(L+\varepsilon)\left(R_{*}+\varepsilon\right) \\
& -\liminf _{t \rightarrow \infty} \frac{1}{R_{1}(t)} \int_{T}^{t} R_{1}^{\alpha+1}(s) \varphi(s) q(s) \Delta s-\alpha\left(r_{*}-\varepsilon\right)^{1+\frac{1}{\alpha}}
\end{aligned}
$$

Since $\varepsilon>0$ is arbitrary, we have the desired inequality:

$$
\liminf _{t \rightarrow \infty} \frac{1}{R_{1}(t)} \int_{T}^{t} R_{1}^{\alpha+1}(s) \varphi(s) q(s) \Delta s \leq[(\alpha+1) L-1] R_{*}-\alpha r_{*}^{1+\frac{1}{\alpha}} .
$$

This completes the proof.

Lemma 3.10 Let $0<\alpha \leq 1$ and $x(t)$ be an eventually positive solution of (1.1) satisfying (I) of Lemma 3.1. Then

$$
\liminf _{t \rightarrow \infty} \frac{1}{R_{1}(t)} \int_{T}^{t} R_{1}^{\alpha+1}(s) \varphi(s) q(s) \Delta s \leq R_{*}(\alpha+L-1)-\alpha r_{*}^{1+\frac{1}{\alpha}}
$$

for sufficiently large $T \in\left[t_{0}, \infty\right)_{\mathbb{T}}$.

Proof As shown in the proof of Lemma 3.9 we have

$$
\begin{aligned}
R_{1}^{\alpha+1}(t) w(t) \leq & R_{1}^{\alpha+1}(T) w(T)+\int_{T}^{t}\left[R_{1}^{\alpha+1}(s)\right]^{\Delta} w(\sigma(s)) \Delta s \\
& -\int_{T}^{t} R_{1}^{\alpha+1}(s) \varphi(s) q(s) \Delta s-\alpha\left(r_{*}-\varepsilon\right)^{1+\frac{1}{\alpha}}\left[R_{1}(t)-R_{1}(T)\right]
\end{aligned}
$$


Assume

$$
\left[R_{1}^{\alpha+1}(s)\right]^{\Delta}=\left[R_{1}^{\alpha}(s) R_{1}(s)\right]^{\Delta}=\left[R_{1}^{\alpha}(s)\right]^{\Delta} R_{1}(s)+R_{1}^{\alpha}(\sigma(s)) R_{1}^{\Delta}(s) .
$$

By the Pötzsche chain rule we obtain

$$
\begin{aligned}
{\left[R_{1}^{\alpha}(s)\right]^{\Delta} } & =\alpha\left(\int_{0}^{1}\left[R_{1}(s)+h \mu(t) R_{1}^{\Delta}(s)\right]^{\alpha-1} d h\right) R_{1}^{\Delta}(s) \\
& =\alpha\left(\int_{0}^{1}\left[(1-h) R_{1}(s)+h R_{1}(\sigma(s))\right]^{\alpha-1} d h\right) R_{1}^{\Delta}(s) \\
& \leq \alpha R_{1}^{\alpha-1}(s) R_{1}^{\Delta}(s) .
\end{aligned}
$$

Then

$$
\begin{aligned}
{\left[R_{1}^{\alpha+1}(s)\right]^{\Delta} } & \leq\left[\alpha R_{1}^{\alpha}(s)+R_{1}^{\alpha}(\sigma(s))\right] R_{1}^{\Delta}(s) \\
& =\left[\alpha R_{1}^{\alpha}(s)+R_{1}^{\alpha}(\sigma(s))\right] r_{1}^{-1 / \alpha_{1}}(s)
\end{aligned}
$$

and so

$$
\begin{aligned}
R_{1}^{\alpha+1}(t) w(t) \leq & R_{1}^{\alpha+1}(T) w(T) \\
& +\int_{T}^{t}\left[\alpha R_{1}^{\alpha}(s)+R_{1}^{\alpha}(\sigma(s))\right] r_{1}^{-1 / \alpha_{1}}(s) w(\sigma(s)) \Delta s \\
& -\int_{T}^{t} R_{1}^{\alpha+1}(s) \varphi(s) q(s) \Delta s-\alpha\left(r_{*}-\varepsilon\right)^{1+\frac{1}{\alpha}}\left[R_{1}(t)-R_{1}(T)\right] \\
= & R_{1}^{\alpha+1}(T) w(T) \\
& +\int_{T}^{t}\left[\alpha+\left[\frac{R_{1}(\sigma(s))}{R_{1}(s)}\right]^{\alpha}\right] r_{1}^{-1 / \alpha_{1}}(s) R_{1}^{\alpha}(s) w(\sigma(s)) \Delta s \\
& -\int_{T}^{t} R_{1}^{\alpha+1}(s) \varphi(s) q(s) \Delta s-\alpha\left(r_{*}-\varepsilon\right)^{1+\frac{1}{\alpha}}\left[R_{1}(t)-R_{1}(T)\right] \\
\leq & R_{1}^{\alpha+1}(T) w(T)+(\alpha+L+\varepsilon)\left(R_{*}+\varepsilon\right)\left[R_{1}(t)-R_{1}(T)\right] \\
& -\int_{T}^{t} R_{1}^{\alpha+1}(s) \varphi(s) q(s) \Delta s-\alpha\left(r_{*}-\varepsilon\right)^{1+\frac{1}{\alpha}}\left[R_{1}(t)-R_{1}(T)\right]
\end{aligned}
$$

Dividing both sides by $R_{1}$, we have

$$
\begin{aligned}
R_{1}^{\alpha}(t) w(t) \leq & \frac{R_{1}^{\alpha+1}(T) w(T)}{R_{1}(t)}+(\alpha+L+\varepsilon)\left(R_{*}+\varepsilon\right)\left[1-\frac{R_{1}(T)}{R_{1}(t)}\right] \\
& -\frac{1}{R_{1}(t)} \int_{T}^{t} R_{1}^{\alpha+1}(s) \varphi(s) q(s) \Delta s-\alpha\left(r_{*}-\varepsilon\right)^{1+\frac{1}{\alpha}}\left[1-\frac{R_{1}(T)}{R_{1}(t)}\right]
\end{aligned}
$$

Taking the lim sup of both sides as $t \rightarrow \infty$ and using (1.2), we get

$$
\begin{aligned}
R_{*} \leq & (\alpha+L+\varepsilon)\left(R_{*}+\varepsilon\right)-\liminf _{t \rightarrow \infty} \frac{1}{R_{1}(t)} \int_{T}^{t} R_{1}^{\alpha+1}(s) \varphi(s) q(s) \Delta s \\
& -\alpha\left(r_{*}-\varepsilon\right)^{1+\frac{1}{\alpha}} .
\end{aligned}
$$


Masan et al. Advances in Difference Equations ( 2017) 2017:111

Page 21 of 28

Since $\varepsilon>0$ is arbitrary, we have the desired inequality:

$$
\liminf _{t \rightarrow \infty} \frac{1}{R_{1}(t)} \int_{T}^{t} R_{1}^{\alpha+1}(s) \varphi(s) q(s) \Delta s \leq R_{*}(\alpha+L-1)-\alpha r_{*}^{1+\frac{1}{\alpha}}
$$

This completes the proof.

Lemma 3.11 Let $\alpha \geq 1$ and $x(t)$ be an eventually positive solution of (1.1) satisfying (I) of Lemma 3.1. Then

$$
\liminf _{t \rightarrow \infty} \frac{1}{R_{1}(t)} \int_{T}^{t} R_{1}^{\alpha+1}(s) \varphi(s) q(s) \Delta s \leq \alpha\left(L R_{*}-r_{*}^{1+\frac{1}{\alpha}}\right) .
$$

Proof As seen in the proof of Lemma 3.9, we obtain

$$
\begin{aligned}
R_{1}^{\alpha+1}(t) w(t) \leq & R_{1}^{\alpha+1}(T) w(T)+\int_{T}^{t}\left[R_{1}^{\alpha+1}(s)\right]^{\Delta} w(\sigma(s)) \Delta s \\
& -\int_{T}^{t} R_{1}^{\alpha+1}(s) \varphi(s) q(s) \Delta s-\alpha\left(r_{*}-\varepsilon\right)^{1+\frac{1}{\alpha}}\left[R_{1}(t)-R_{1}(T)\right] .
\end{aligned}
$$

Assume

$$
\left[R_{1}^{\alpha+1}(s)\right]^{\Delta}=\left[R_{1}^{\alpha}(s) R_{1}(s)\right]^{\Delta}=\left[R_{1}^{\alpha}(s)\right]^{\Delta} R_{1}(\sigma(s))+R_{1}^{\alpha}(s) R_{1}^{\Delta}(s) .
$$

By the Pötzsche chain rule, we obtain

$$
\begin{aligned}
{\left[R_{1}^{\alpha}(s)\right]^{\Delta} } & =\alpha\left(\int_{0}^{1}\left[R_{1}(s)+h \mu(t) R_{1}^{\Delta}(s)\right]^{\alpha-1} d h\right) R_{1}^{\Delta}(s) \\
& =\alpha\left(\int_{0}^{1}\left[(1-h) R_{1}(s)+h R_{1}(\sigma(s))\right]^{\alpha-1} d h\right) R_{1}^{\Delta}(s) \\
& \leq \alpha R_{1}^{\alpha-1}(\sigma(s)) R_{1}^{\Delta}(s) .
\end{aligned}
$$

Then

$$
\begin{aligned}
{\left[R_{1}^{\alpha+1}(s)\right]^{\Delta} } & \leq\left[\alpha R_{1}^{\alpha}(\sigma(s))+R_{1}^{\alpha}(s)\right] R_{1}^{\Delta}(s) \\
& =\left[\alpha R_{1}^{\alpha}(\sigma(s))+R_{1}^{\alpha}(s)\right] r_{1}^{-1 / \alpha_{1}}(s)
\end{aligned}
$$

and so

$$
\begin{aligned}
R_{1}^{\alpha+1}(t) w(t) \leq & R_{1}^{\alpha+1}(T) w(T) \\
& +\int_{T}^{t}\left[\alpha R_{1}^{\alpha}(\sigma(s))+R_{1}^{\alpha}(s)\right] r_{1}^{-1 / \alpha_{1}}(s) w(\sigma(s)) \Delta s \\
& -\int_{T}^{t} R_{1}^{\alpha+1}(s) \varphi(s) q(s) \Delta s-\alpha\left(r_{*}-\varepsilon\right)^{1+\frac{1}{\alpha}}\left[R_{1}(t)-R_{1}(T)\right] \\
= & R_{1}^{\alpha+1}(T) w(T) \\
& +\int_{T}^{t}\left[\alpha \frac{R_{1}^{\alpha}(\sigma(s))}{R_{1}^{\alpha}(s)}+1\right] r_{1}^{-1 / \alpha_{1}}(s) R_{1}^{\alpha}(s) w(\sigma(s)) \Delta s
\end{aligned}
$$




$$
\begin{aligned}
& -\int_{T}^{t} R_{1}^{\alpha+1}(s) \varphi(s) q(s) \Delta s-\alpha\left(r_{*}-\varepsilon\right)^{1+\frac{1}{\alpha}}\left[R_{1}(t)-R_{1}(T)\right] \\
\leq & R_{1}^{\alpha+1}(T) w(T)+(\alpha(L+\varepsilon)+1)\left(R_{*}+\varepsilon\right)\left[R_{1}(t)-R_{1}(T)\right] \\
& -\int_{T}^{t} R_{1}^{\alpha+1}(s) \varphi(s) q(s) \Delta s-\alpha\left(r_{*}-\varepsilon\right)^{1+\frac{1}{\alpha}}\left[R_{1}(t)-R_{1}(T)\right] .
\end{aligned}
$$

Dividing both sides by $R_{1}$, we have

$$
\begin{aligned}
R_{1}^{\alpha}(t) w(t) \leq & \frac{R_{1}^{\alpha+1}(T) w(T)}{R_{1}(t)}+(\alpha(L+\varepsilon)+1)\left(R_{*}+\varepsilon\right)\left[1-\frac{R_{1}(T)}{R_{1}(t)}\right] \\
& -\frac{1}{R_{1}(t)} \int_{T}^{t} R_{1}^{\alpha+1}(s) \varphi(s) q(s) \Delta s \\
& -\alpha\left(r_{*}-\varepsilon\right)^{1+\frac{1}{\alpha}}\left[1-\frac{R_{1}(T)}{R_{1}(t)}\right] .
\end{aligned}
$$

Taking the lim sup of both sides as $t \rightarrow \infty$ and using (1.2), we get

$$
\begin{aligned}
R_{*} \leq & (\alpha(L+\varepsilon)+1)\left(R_{*}+\varepsilon\right) \\
& -\liminf _{t \rightarrow \infty} \frac{1}{R_{1}(t)} \int_{T}^{t} R_{1}^{\alpha+1}(s) \varphi(s) q(s) \Delta s-\alpha\left(r_{*}-\varepsilon\right)^{1+\frac{1}{\alpha}} .
\end{aligned}
$$

Since $\varepsilon>0$ is arbitrary, we have the desired inequality:

$$
\liminf _{t \rightarrow \infty} \frac{1}{R_{1}(t)} \int_{T}^{t} R_{1}^{\alpha+1}(s) \varphi(s) q(s) \Delta s \leq \alpha\left(L R_{*}-r_{*}^{1+\frac{1}{\alpha}}\right) .
$$

This completes the proof.

Remark 3.1 The conclusion of Lemmas 3.5-3.11 remains intact if $\varphi(1.1)$ is replaced by $\bar{\varphi}$.

\section{Proofs of the main results}

In this section we prove the main results.

Proof of Theorem 2.1 Assume equation (1.1) has a nonoscillatory solution $x$ on $\left[t_{0}, \infty\right)_{\mathbb{T}}$. Then, without loss of generality, assume $x(t)>0$ and $x(g(t))>0$ on $\left[t_{0}, \infty\right)_{\mathbb{T}}$. Then if case (I) of Lemma 3.1 holds, so by Lemma 3.3, we see

$$
\int_{t_{0}}^{\infty} q(s) \Delta s<\infty
$$

which contradicts (2.1). Now if case (II) of Lemma 3.1 holds, then by Lemma 3.2, we get $\lim _{t \rightarrow \infty} x(t)=0$. The proof is complete.

Proof of Theorem 2.2 Assume equation (1.1) has a nonoscillatory solution $x$ on $\left[t_{0}, \infty\right)_{\mathbb{T}}$. Then, without loss of generality, assume $x(t)>0$ and $x(g(t))>0$ on $\left[t_{0}, \infty\right)_{\mathbb{T}}$. Then if case (I) of Lemma 3.1 holds, so by Lemma 3.5, we see

$$
\int_{t_{0}}^{\infty} \phi(s) q(s) \Delta s<\infty
$$


which contradicts (2.3). Now if case (II) of Lemma 3.1 holds, then by Lemma 3.2, we get $\lim _{t \rightarrow \infty} x(t)=0$. The proof is complete.

Proof of Theorem 2.3 Assume equation (1.1) has a nonoscillatory solution $x$ on $\left[t_{0}, \infty\right)_{\mathbb{T}}$. Then, without loss of generality, assume $x(t)>0$ and $x(g(t))>0$ on $\left[t_{0}, \infty\right)_{\mathbb{T}}$. Then if case (I) of Lemma 3.1 holds, then by Lemma 3.7, we see

$$
\liminf _{t \rightarrow \infty} R_{1}^{\alpha}(t) \int_{\sigma(t)}^{\infty} \varphi(s) q(s) \Delta s \leq r_{*}-\frac{r_{*}^{1+\frac{1}{\alpha}}}{L} .
$$

Using the inequality (3.14) with $A=\frac{1}{L}, B=1$ and $u=r_{*}$, we get the desired inequality:

$$
\liminf _{t \rightarrow \infty} R_{1}^{\alpha}(t) \int_{\sigma(t)}^{\infty} \varphi(s) q(s) \Delta s \leq \frac{(\alpha L)^{\alpha}}{(\alpha+1)^{\alpha+1}}
$$

which contradicts (2.5). Now if case (II) of Lemma 3.1 holds, then by Lemma 3.2, we get $\lim _{t \rightarrow \infty} x(t)=0$. The proof is complete.

Proof of Theorem 2.4 Assume equation (1.1) has a nonoscillatory solution $x$ on $\left[t_{0}, \infty\right)_{\mathbb{T}}$. Then, without loss of generality, assume $x(t)>0$ and $x(g(t))>0$ on $\left[t_{0}, \infty\right)_{\mathbb{T}}$. Then if case (I) of Lemma 3.1 holds, then by Lemmas 3.8 and 3.9, we have

$$
R_{*} \leq L^{\alpha+1}-\liminf _{t \rightarrow \infty} \frac{1}{R_{1}(t)} \int_{t_{2}}^{t} R_{1}^{\alpha+1}(s) \varphi(s) q(s) \Delta s
$$

and

$$
\liminf _{t \rightarrow \infty} \frac{1}{R_{1}(t)} \int_{t_{2}}^{t} R_{1}^{\alpha+1}(s) \varphi(s) q(s) \Delta s \leq R_{*}[(\alpha+1) L-1]-\alpha r_{*}^{1+\frac{1}{\alpha}}
$$

Therefore

$$
\liminf _{t \rightarrow \infty} \frac{1}{R_{1}(t)} \int_{t_{2}}^{t} R_{1}^{\alpha+1}(s) \varphi(s) q(s) \Delta s \leq L^{\alpha+1}\left(1-\frac{1}{(\alpha+1) L}\right)
$$

which contradicts the condition (2.7). If case (II) of Lemma 3.1 holds, then by Lemma 3.2, we get $\lim _{t \rightarrow \infty} x(t)=0$. The proof is complete.

Proof of Theorem 2.5 Assume equation (1.1) has a nonoscillatory solution $x$ on $\left[t_{0}, \infty\right)_{\mathbb{T}}$. Then, without loss of generality, assume $x(t)>0$ and $x(g(t))>0$ on $\left[t_{0}, \infty\right)_{\mathbb{T}}$. Then if case (I) of Lemma 3.1 holds, then by Lemmas 3.8 and 3.10, we have

$$
R_{*} \leq L^{\alpha+1}-\liminf _{t \rightarrow \infty} \frac{1}{R_{1}(t)} \int_{t_{2}}^{t} R_{1}^{\alpha+1}(s) \varphi(s) q(s) \Delta s
$$

and

$$
\liminf _{t \rightarrow \infty} \frac{1}{R_{1}(t)} \int_{t_{2}}^{t} R_{1}^{\alpha+1}(s) \varphi(s) q(s) \Delta s \leq R_{*}[\alpha+L-1]-\alpha r_{*}^{1+\frac{1}{\alpha}}
$$


Therefore

$$
\liminf _{t \rightarrow \infty} \frac{1}{R_{1}(t)} \int_{t_{2}}^{t} R_{1}^{\alpha+1}(s) \varphi(s) q(s) \Delta s \leq L^{\alpha+1}\left(1-\frac{1}{\alpha+L}\right),
$$

which contradicts the condition (2.9). If case (II) of Lemma 3.1 holds, then by Lemma 3.2, we get $\lim _{t \rightarrow \infty} x(t)=0$. The proof is complete.

Proof of Theorem 2.6 Assume equation (1.1) has a nonoscillatory solution $x$ on $\left[t_{0}, \infty\right)_{\mathbb{T}}$. Then, without loss of generality, assume $x(t)>0$ and $x(g(t))>0$ on $\left[t_{0}, \infty\right)_{\mathbb{T}}$. Then if case (I) of Lemma 3.1 holds, then by Lemmas 3.8 and 3.11, we have

$$
R_{*} \leq L^{\alpha+1}-\liminf _{t \rightarrow \infty} \frac{1}{R_{1}(t)} \int_{t_{2}}^{t} R_{1}^{\alpha+1}(s) \varphi(s) q(s) \Delta s
$$

and

$$
\liminf _{t \rightarrow \infty} \frac{1}{R_{1}(t)} \int_{T}^{t} R_{1}^{\alpha+1}(s) \varphi(s) q(s) \Delta s \leq \alpha\left(L R_{*}-r_{*}^{\frac{\alpha+1}{\alpha}}\right) .
$$

Thus

$$
\liminf _{t \rightarrow \infty} \frac{1}{R_{1}(t)} \int_{t_{2}}^{t} R_{1}^{\alpha+1}(s) \varphi(s) q(s) \Delta s \leq \frac{\alpha L^{\alpha+2}}{1+\alpha L},
$$

which contradicts the condition (2.10). If case (II) of Lemma 3.1 holds, then by Lemma 3.2, we get $\lim _{t \rightarrow \infty} x(t)=0$. The proof is complete.

Proof of Theorem 2.7 Assume equation (1.1) has a nonoscillatory solution $x$ on $\left[t_{0}, \infty\right)_{\mathbb{T}}$. Then, without loss of generality, assume $x(t)>0$ and $x(g(t))>0$ on $\left[t_{0}, \infty\right)_{\mathbb{T}}$. Then if case (I) of Lemma 3.1 holds, then

$$
x^{[1]}(t)>0, \quad x^{[2]}(t)>0, \quad\left[x^{[2]}(t)\right]^{\Delta}<0 \quad \text { on }\left[t_{0}, \infty\right)_{\mathbb{T}} .
$$

Integrating both sides of the dynamic equation (1.1) from $t$ to $v \in\left[t_{0}, \infty\right)_{\mathbb{T}}$, we obtain

$$
\int_{t}^{v} q(s) x^{\alpha}(g(s)) \Delta s=x^{[2]}(t)-x^{[2]}(v) \leq x^{[2]}(t) .
$$

As shown in the proof of Lemmas 3.4 and 3.5, we have, for $t \geq t_{1}$,

$$
x^{\alpha}(t) \geq x^{[2]}(t) R_{3}^{\alpha}(t)
$$

and

$$
x^{\alpha}(g(t)) \geq \varphi(t) x^{\alpha}(t)
$$

for some $t_{1} \in\left(t_{0}, \infty\right)_{\mathbb{T}}$ such that $g(t) \in\left(t_{0}, \infty\right)_{\mathbb{T}}$ for $t \geq t_{1}$. From (4.1) and (4.3), we obtain

$$
\int_{t}^{v} \varphi(s) q(s) x^{\alpha}(s) \Delta s \leq x^{[2]}(t)
$$


Since $x^{\Delta}(t)>0$, we get

$$
x^{\alpha}(t) \int_{t}^{v} \varphi(s) q(s) \Delta s \leq x^{[2]}(t)
$$

From (4.2) and (4.4), we get

$$
R_{3}^{\alpha}(t) \int_{t}^{v} \varphi(s) q(s) \Delta s \leq 1 .
$$

Taking $v \rightarrow \infty$, we have

$$
R_{3}^{\alpha}(t) \int_{t}^{\infty} \varphi(s) q(s) \Delta s \leq 1,
$$

which gives us the contradiction

$$
\limsup _{t \rightarrow \infty} R_{3}^{\alpha}(t) \int_{t}^{\infty} \varphi(s) q(s) \Delta s \leq 1 .
$$

Now if case (II) of Lemma 3.1 holds, then by Lemma 3.2, we get $\lim _{t \rightarrow \infty} x(t)=0$. The proof is complete.

Proof of Theorem 2.8 The proof is similar to that of previous results where $\varphi$ is replaced by $\bar{\varphi}$; see Lemma 3.6 and Remark 3.1.

Proof of Theorem 2.9 Assume equation (1.1) has a nonoscillatory solution $x$ on $\left[t_{0}, \infty\right)_{\mathbb{T}}$. Then, without loss of generality, assume $x(t)>0$ and $x(g(t))>0$ on $\left[t_{0}, \infty\right)_{\mathbb{T}}$. Then if case (I) of Lemma 3.1 holds, then

$$
x^{[1]}(t)>0, \quad x^{[2]}(t)>0, \quad\left[x^{[2]}(t)\right]^{\Delta}<0 \quad \text { on }\left[t_{0}, \infty\right)_{\mathbb{T}} .
$$

Define

$$
z(t):=\frac{x^{[2]}(t)}{\left(x^{[1]}(t)\right)^{\alpha_{2}}} .
$$

By the product rule and the quotient rule, we get

$$
\begin{aligned}
z^{\Delta}(t) & =\frac{1}{\left(x^{[1]}(t)\right)^{\alpha_{2}}}\left(x^{[2]}(t)\right)^{\Delta}+\left(\frac{1}{\left(x^{[1]}(t)\right)^{\alpha_{2}}}\right)^{\Delta} x^{[2]}(\sigma(t)) \\
& =\frac{\left(x^{[2]}(t)\right)^{\Delta}}{\left(x^{[1]}(t)\right)^{\alpha_{2}}}-\frac{\left(\left(x^{[1]}(t)\right)^{\alpha_{2}}\right)^{\Delta}}{\left(x^{[1]}(t)\right)^{\alpha_{2}}\left(x^{[1]}(\sigma(t))\right)^{\alpha_{2}}} x^{[2]}(\sigma(t)) .
\end{aligned}
$$

From (1.1) and the definition of $z(t)$, we see that, for $t \geq t_{0}$,

$$
z^{\Delta}(t)=-q(t) \frac{x^{\alpha}(g(t))}{\left(x^{[1]}(t)\right)^{\alpha_{2}}}-\frac{\left(\left(x^{[1]}(t)\right)^{\alpha_{2}}\right)^{\Delta}}{\left(x^{[1]}(t)\right)^{\alpha_{2}}} z(\sigma(t)) .
$$

Hence

$$
\frac{x^{\alpha}(g(t))}{\left(x^{[1]}(t)\right)^{\alpha_{2}}}=\left[\frac{x(g(t))}{x(t)}\right]^{\alpha} \frac{x^{\alpha}(t)}{\left(x^{[1]}(t)\right)^{\alpha_{2}}} .
$$


As shown in the proof of Lemma 3.4 we get

$$
\frac{x^{\alpha}(t)}{\left(x^{[1]}(t)\right)^{\alpha_{2}}} \geq R^{\alpha}(t)
$$

and as in the proof of Lemma 3.5, there exists $t_{1} \in\left[t_{0}, \infty\right)_{\mathbb{T}}$ such that $g(t)>t_{0}$ and

$$
\left[\frac{x(g(t))}{x(t)}\right]^{\alpha} \geq \varphi(t)
$$

Then

$$
\frac{x^{\alpha}(g(t))}{\left(x^{[1]}(t)\right)^{\alpha_{2}}} \geq \hat{\varphi}(t)
$$

It follows that, for $t \geq t_{1}$,

$$
z^{\Delta}(t) \leq-\hat{\varphi}(t) q(t)-\frac{\left(\left(x^{[1]}(t)\right)^{\alpha_{2}}\right)^{\Delta}}{\left(x^{[1]}(t)\right)^{\alpha_{2}}} z(\sigma(t))
$$

By the Pötzsche chain rule,

$$
\left(\left(x^{[1]}(t)\right)^{\alpha_{2}}\right)^{\Delta} \geq \begin{cases}\alpha_{2}\left[x^{[1]}(\sigma(t))\right]^{\alpha_{2}-1}\left(x^{[1]}(t)\right)^{\Delta}, & 0<\alpha_{2} \leq 1 \\ \alpha_{2}\left[x^{[1]}(t)\right]^{\alpha_{2}-1}\left(x^{[1]}(t)\right)^{\Delta}, & \alpha_{2} \geq 1\end{cases}
$$

If $0<\alpha_{2} \leq 1$, we have

$$
z^{\Delta}(t) \leq-\hat{\varphi}(t) q(t)-\frac{\alpha_{2} z(\sigma(t))}{\rho(\sigma(t))} \frac{\left(x^{[1]}(t)\right)^{\Delta}}{x^{[1]}(\sigma(t))}\left(\frac{x^{[1]}(\sigma(t))}{x^{[1]}(t)}\right)^{\alpha_{2}}
$$

and if $\alpha_{2} \geq 1$, we have

$$
z^{\Delta}(t) \leq-Q_{2}(t)-\alpha_{2} \frac{\left(x^{[1]}(t)\right)^{\Delta}}{x^{[1]}(\sigma(t))} \frac{x^{[1]}(\sigma(t))}{x^{[1]}(t)} z(\sigma(t))
$$

Since $x^{[1]}$ is strictly increasing and $x^{[2]}$ is strictly decreasing, we get

$$
x^{[1]}(\sigma(t)) \geq x^{[1]}(t) \text { and }\left(x^{[1]}(t)\right)^{\Delta} \geq\left(\frac{x^{[2]}(\sigma(t))}{r_{2}(t)}\right)^{1 / \alpha_{2}}
$$

Then from (4.5) and (4.6) we obtain

$$
z^{\Delta}(t) \leq-\hat{\varphi}(t) q(t)-\alpha_{2} r_{2}^{-1 / \alpha_{2}}(t) z^{1+\frac{1}{\alpha_{2}}}(\sigma(t)) \quad \text { for } t \geq t_{1}
$$

The rest of the argument is similar to that of previous results with $R_{1}$ is replaced by $R_{2}$ and hence is omitted.

The authors declare that they have no competing interests. 


\section{Author details}

${ }^{1}$ Department of Mathematics, Faculty of Science, University of Hail, Hail, 2440, Saudi Arabia. ${ }^{2}$ Department of Mathematics, Faculty of Science, Mansoura University, Mansoura, 35516, Egypt. ${ }^{3}$ Department of Mathematics, Texas A\&M University-Kingsville, 700 University Blvd., Kingsville, TX 78363-8202, USA. ${ }^{4}$ Nonlinear Analysis and Applied Mathematics Research Group (NAAM), King Abdulaziz University, P.O. Box 80203, Jeddah, 21589, Saudi Arabia.

\section{Acknowledgements}

The authors would like to thank the referees for their careful reading of the entire manuscript, which helped to significantly improve the quality of this paper. This work was supported by Research Deanship of Hail University under grant No. 0150258.

\section{Publisher's Note}

Springer Nature remains neutral with regard to jurisdictional claims in published maps and institutional affiliations.

Received: 14 December 2016 Accepted: 31 March 2017 Published online: 13 April 2017

\section{References}

1. Bohner, M, Peterson, A: Dynamic Equations on Time Scales: An Introduction with Applications. Birkhäuser, Boston (2001)

2. Hilger, S: Analysis on measure chains - a unified approach to continuous and discrete calculus. Results Math. 18 18-56 (1990)

3. Fite, WB: Concerning the zeros of the solutions of certain differential equations. Trans. Am. Math. Soc. 19, 341-352 (1918)

4. Hille, E: Non-oscillation theorems. Trans. Am. Math. Soc. 64, 234-252 (1948)

5. Nehari, Z: Oscillation criteria for second-order linear differential equations. Trans. Am. Math. Soc. 85, 428-445 (1957)

6. Wong, JSW: Second order oscillation with retarded arguments. In: Ordinary Differential Equations, pp. 581-596. Academic Press, New York (1972)

7. Erbe, L: Oscillation criteria for second order nonlinear delay equations. Can. Math. Bull. 16, 49-56 (1973)

8. Ohriska, J: Oscillation of second order delay and ordinary differential equations. Czechoslov. Math. J. 34, 107-112 (1984)

9. Erbe, L, Peterson, A, Saker, SH: Hille and Nehari type criteria for third-order dynamic equations. J. Math. Anal. Appl. 329, 112-131 (2007)

10. Erbe, L, Hassan, TS, Peterson, A: Oscillation of third-order nonlinear functional dynamic equations on time scales. Differ. Equ. Dyn. Syst. 18, 199-227 (2010)

11. Saker, SH: Oscillation of third-order functional dynamic equations on time scales. Sci. China Math. 54(12), 2597-2614 (2011)

12. Wang, $Y, X u, Z$ : Asymptotic properties of solutions of certain third-order dynamic equations. J. Comput. Appl. Math 236, 2354-2366 (2012)

13. Agarwal, RP, Bohner, M, Li, T, Zhang, C: Hille and Nehari type criteria for third-order delay dynamic equations. J. Differ. Equ. Appl. 19, 1563-1579 (2013)

14. Anderson, DR: Oscillation and nonoscillation criteria for two-dimensional time scale systems of first order nonlinear dynamic equations. Electron. J. Differ. Equ. 2009, Article ID 24 (2009)

15. Erbe, L, Hassan, TS, Peterson, A: Oscillation criteria for nonlinear damped dynamic equations on time scales. Appl. Math. Comput. 203, 343-357 (2008)

16. Erbe, L, Hassan, TS, Peterson, A: Oscillation criteria for nonlinear functional neutral dynamic equations on time scales. J. Differ. Equ. Appl. 15, 1097-1116 (2009)

17. Erbe, L, Hassan, TS, Peterson, A: Oscillation of second order functional dynamic equations. Int. J. Differ. Equ. Appl. 5(2), 175-193 (2010)

18. Hassan, TS: Oscillation criteria for half-linear dynamic equations on time scales. J. Math. Anal. Appl. 345, 176-185 (2008)

19. Erbe, L, Hassan, TS, Peterson, A, Saker, SH: Oscillation criteria for sublinear half-linear delay dynamic equations on time scales. Int. J. Difference Equ. 3, 227-245 (2008)

20. Erbe, L, Hassan, TS, Peterson, A, Saker, SH: Oscillation criteria for half-linear delay dynamic equations on time scales. Nonlinear Dyn. Syst. Theory 9(1), 51-68 (2009)

21. Hassan, TS: Oscillation of third-order nonlinear delay dynamic equations on time scales. Math. Comput. Model. 49 1573-1586 (2009)

22. Han, Z, Li, T, Sun, S, Zhang, M: Oscillation behavior of solutions of third-order nonlinear delay dynamic equations on time scales. Commun. Korean Math. Soc. 26, 499-513 (2011)

23. Li, T, Han, Z, Sun, S, Zhao, Y: Oscillation results for third-order nonlinear delay dynamic equations on time scales. Bull. Malays. Math. Soc. 34, 639-648 (2011)

24. Li, T, Han, Z, Sun, Y, Zhao, Y: Asymptotic behavior of solutions for third-order half-linear delay dynamic equations on time scales. J. Appl. Math. Comput. 36, 333-346 (2011)

25. Hovhannisy, G: On oscillations of solutions of third-order dynamic equation. Abstr. Appl. Anal. 2012, Article ID 71598 (2012)

26. Senel, MT: Behavior of solutions of a third-order dynamic equation on time scales. J. Inequal. Appl. 2013, Article ID 47 (2013)

27. Sun, Y, Han, Z, Sun, Y, Pan, Y: Oscillation theorems for certain third-order nonlinear delay dynamic equations on time scales. Electron. J. Qual. Theory Differ. Equ. 2011, Article ID 75 (2011)

28. Yu, Z, Wang, Q: Asymptotic behavior of solutions of third-order dynamic equations on time scales. J. Comput. Appl. Math. 255, 531-540 (2009) 
29. Bohner, M, Grace, SR, Sağer, I, Tunç, E: Oscillation of third-order nonlinear damped delay differential equations. Appl. Math. Comput. 278, 21-32 (2016)

30. Grace, SR, Graef, JR, Tunç, E: Oscillatory behavior of a third-order neutral dynamic equation with distributed delays. Electron. J. Qual. Theory Differ. Equ. 2016, Article ID 14 (2016). Proceedings of the 10th Colloquium on the Qualitative Theory of Differential Equations (July 1-4, 2015, Szeged, Hungary)

Submit your manuscript to a SpringerOpen ${ }^{\circ}$ journal and benefit from:

- Convenient online submission

- Rigorous peer review

- Immediate publication on acceptance

- Open access: articles freely available online

- High visibility within the field

- Retaining the copyright to your article 